\title{
THE BIRATIONAL GEOMETRY OF UNRAMIFIED IRREGULAR HIGGS BUNDLES ON CURVES
}

\author{
SZILÁRD SZABÓ \\ BUDAPEST UNIVERSITY OF TECHNOLOGY AND ECONOMICS, \\ EGRY J. U. 1., 1111 BUDAPEST, HUNGARY \\ SZABOSZ@MATH.BME.HU
}

\begin{abstract}
We give a variant of the Beauville-Narasimhan-Ramanan correspondence for irregular parabolic Higgs bundles on smooth projective curves with fixed semi-simple irregular part and show that it defines a Poisson isomorphism between certain irregular Dolbeault moduli spaces and relative Picard bundles of families of ruled surfaces over the curve.
\end{abstract}

\section{INTRODUCTION}

The Beauville-Narasimhan-Ramanan (BNR) correspondence [4] provides an equivalence of categories between (an open subset of) the category of twisted Higgs bundles $(\mathcal{E}, \theta)$ over a smooth projective curve and torsionfree sheaves $S$ of rank 1 on integral finite covers of the curve contained in a ruled surface $Z$ and intersecting the infinity-divisor trivially. The functor simply turns the action of the Higgs field into the action of multiplication by a variable algebraically independent from the function field of the curve; regularity of the Higgs field amounts to saying that we obtain the action of a commutative algebra. This correspondence is also closely related to Hitchin's integrable system [12], namely the description of an open subset of the moduli space of stabe Higgs bundles over a curve in terms of its spectral cover and spectral sheaf. The Hitchin map associates to a given Higgs bundle the characteristic coefficients of the Higgs field and the generic fiber is the Jacobi-variety of the corresponding smooth curve. The setup of the original BNR correspondence has been generalized to the case of arbitrary (not necessarily integral) spectral schemes by D. Schaub [21].

Mehta and Seshadri [19] generalized the notion of holomorphic vector bundle over a curve to the notion of parabolic vector bundle over a curve with marked points. Higgs bundles with tame (or regular) singularities compatible with a parabolic structure at the marked points have first been studied by C. Simpson [22]. In [26] K. Yokogawa and in [9] H. Boden

Date: February 22, 2017. 
and K. Yokogawa constructed a moduli space of parabolic Higgs bundles with regular singularities using Geometric Invariant Theory; parallelly, a gauge-theoretic construction was provided by H. Konno [15]. Later, Higgs bundles with wild (or irregular) singularities endowed with compatible parabolic structures have also been extensively studied from various perspectives in the mathematical literature (see e.g. O. Biquard and P. Boalch [5], P. Boalch [8], T. Mochizuki [20]). They equally showed up in the physics literature c.f. E. Witten [25], due to their role in electric-magnetic duality. The moduli spaces of stable Higgs bundles with (tame or wild) singularities at prescribed marked points on a fixed smooth projective curve were proven to be holomorphic Poisson varieties in case one allows the singular parts of the Higgs field vary by E. Markman [18], and holomorphic symplectic (actually, complete hyperKähler) manifolds in case one requires the singular parts to be equivalent to given semi-simple local forms [5].

As the original BNR-correpondence holds for twisted Higgs bundles, it is valid in particular for Higgs bundles with arbitrary singularities. It allows one to determine explicitly some holomorphic Poisson moduli spaces of stable parabolic Higgs bundles. However, for explicit computation of symplectic leaves of these holomorphic Poisson spaces, namely moduli spaces of stable parabolic Higgs bundles with fixed semi-simple singular parts, it would be useful to have a refined correspondence that takes into account the fixing of the singular parts too. Such a refined version in the case of regular (or logarithmic) singularities has been provided by M. Logares and J. Martens [17]. However, to our knowledge such a refined BNR-construction in the irregular singular case has not yet been worked out; the purpose of this paper is to give such a refinement. The route we take is slightly different from Logares and Martens even in the logarithmic case, in that we encode the parabolic structure of the bundle in a (R-)parabolic structure of the spectral sheaf. Furthermore, as it was pointed out in Section 4 of [1] by K. Aker and the author, for such a correspondence in the irregular parabolic case one needs to perform iterated blow-ups along non-reduced 0-dimensional subschemes of the fibres of $Z$ over the irregular singular points. In addition, the spectral sheaves transform by a so-called proper transform functor for coherent sheaves, whose definition and main properties are given in Section 5. loc. cit. Our approach here will be parallel to (but more direct than) the one developed in [1].

Motivated by Mirror Symmetry, M. Kontsevich and Y. Soibelman sketch the idea of multiple blow-ups according to some configurations prescribed by the local singular types in Section 8.3 of [16] (without however underlining the role of the parabolic structure on the spectral data). Their analysis is reminiscent to the work of R. Donagi and E. Markman [10], where the symplectic leaves of the holomorphic Poisson manifold of certain rank 1 
sheaves supported purely in dimension 1 over a Poisson surface are determined to be given by fixing the intersection of the support curves with the degeneracy divisor of the Poisson structure. Our aim in this paper is to fill out the details of this correspondence. In particular, our results imply part 1) of Conjecture 8.6.1 of [16] in the semi-simple case. Specifically, we will show the following.

Theorem 1.1. Given a smooth complex projective curve $C$, a finite set $p_{1}, \ldots, p_{n} \in C$ and multiplicities $m_{i} \in \mathbf{N}$ for $1 \leq i \leq n$, there exists a family of ruled surfaces

$$
\widetilde{\mathcal{Z}} \rightarrow \mathbf{C}^{r \sum_{i}\left(m_{i}+1\right)}
$$

over $C$ such that there exists a Poisson isomorphism between dense open subsets of the moduli space of stable irregular Higgs bundles on $C$ with semi-simple irregular parts at $p_{i}$ with pole of order $m_{i}+1$ and no other singularity on the one hand, and on the other hand the relative Picard bundle of certain torsion-free sheaves of rank 1 over a relative Hilbert scheme of curves in $\widetilde{\mathcal{Z}}$.

In concrete terms, the open subset of the moduli space of stable Higgs bundles appearing in the Theorem is characterised by the condition that the spectral curve be smooth. For a more precise statement of the result see Theorem 5.4.

The key ingredient in the proof of the above theorem is an equivalence of categories in the same spirit proven in Theorem 4.3 for objects endowed with a compatible parabolic structure. Theorem 4.3 holds under a milder assumption on the spectral curve than Theorem 5.4, namely integrality. Once this categorical correspondence is established, it is a relatively routine matter to deduce Theorem 5.4. Actually, as the Poisson spaces of sheaves considered in Theorem 5.4 are birational to the Poisson spaces considered by Markman [18], Theorem 5.4 directly follows from Theorem 4.3 coupled with Corollary 7.15 of [18]. See also [13] and [11] where similar Poisson isomorphism statements are proven at various levels of generality. Nevertheless, we include a derivation of Theorem 5.4 in the spirit of [24] for the sake of completeness.

Let us also point out that there exists a different characterization of an open subset of moduli spaces of stable Higgs bundles with irregular singularities using quiver varieties. The advantage of our approach as opposed to the one using quivers is that our description is not limited to Higgs bundles on holomorphically trivial line bundles.

The paper is organized as follows. In Section 2 we set some notation concerning irregular Higgs bundles and parabolic sheaves. In Section 3 we give the correspondence in the case of Higgs bundles with regular singularities (Proposition 3.2). Then we turn to the irregular case in Section 4 and 
extend the correspondence to this case in Theorem 4.3. Finally, in Section 5 we spell out the natural Poisson structures on the relative Dolbeault moduli space and the relative Picard bundle and show that the correspondence is a Poisson isomorphism between dense open subsets (Theorem 5.4).

\section{IrRegular PARABOlic Higgs BundLeS AND PARABOLIC SHEAVES}

In this section we will introduce some basic terminology on the one hand concerning irregular Higgs bundles on curves along the lines of P. Boalch's paper [7] and on the other hand concerning parabolic sheaves, and establish some elementary results about these objects. We end the section with a brief description of a ruled surface.

2.1. Irregular parabolic Higgs bundles. Let $C$ be a smooth complex projective curve over $\mathbf{C}$ and $\mathcal{E}$ a holomorphic vector bundle of rank $r \geq 2$ over $C$. We denote by $\mathcal{O}_{C}, K_{C}$ the regular and canonical sheaves of $C$ respectively and by $\mathrm{d}$ the differential of meromorphic forms on $C$. Let us fix finitely many points $p_{1}, \ldots, p_{n} \in C$ and non-negative integers $m_{1}, \ldots, m_{n} \in$ $\mathbf{N}$. For each $i$ we also fix a local coordinate $z_{i}$ of $C$ near $p_{i}$ and an irregular part

$$
Q_{i}=A_{i}^{m_{i}} z_{i}^{-m_{i}}+\cdots+A_{i}^{1} z_{i}^{-1}
$$

where $A_{i}^{j} \in \mathfrak{g l}_{r}(\mathbf{C})$ are semi-simple endomorphisms satisfying

$$
\left[A_{i}^{j}, A_{i}^{j^{\prime}}\right]=0 \text {. }
$$

In (1) the exponents of $z_{i}$ are meant to increase from left to right; in the particular case $m_{i}=0$ the irregular part is $Q_{i}=0$, and in this case we say that $\theta$ has regular or logarithmic singularities. We set

$$
D=\left(m_{1}+1\right) \cdot p_{1}+\cdots+\left(m_{n}+1\right) \cdot p_{n}
$$

as an effective divisor on $C$ with associated reduced divisor

$$
D_{\text {red }}=p_{1}+\cdots+p_{n} \text {. }
$$

Moreover we set

$$
L=K_{C}(D) .
$$

By (untwisted) irregular Higgs field with local form (1) we mean a section

$$
\theta \in H^{0}\left(C, \mathcal{E} n d(\mathcal{E}) \otimes_{\mathcal{O}_{C}} L\right)
$$

such that for any $i \in\{1, \ldots, n\}$ there exists a local trivialisation of $\mathcal{E}$ near $p_{i}$ with respect to which we have an expansion

$$
\theta=\mathrm{d} Q_{i}+\left(\Lambda_{i} z_{i}^{-1}+\text { holomorphic terms }\right) \mathrm{d} z_{i}
$$


for some $\Lambda_{i} \in \mathfrak{g l}_{r}(\mathbf{C})$. A quasi-parabolic structure compatible with $\theta$ at $p_{i}$ is a filtration

$$
\{0\} \subset F_{i}^{l_{i}-1} \subset \cdots \subset F_{i}^{1} \subset F_{i}^{0}=\mathcal{E}_{\mid p_{i}}
$$

of some length $1 \leq l_{i} \leq r$ preserved by the matrix $\Lambda_{i}$ and the irregular part $Q_{i}$ (i.e. by all the matrices $A_{i}^{1}, \ldots, A_{i}^{m_{i}}$ ).

Claim 2.1. $Q_{i}$ preserves the filtration (4) if and only if for any $l$ the piece $F_{i}^{l}$ in (4) is the direct sum of its intersections with the various simultaneous eigenspaces of $A_{i}^{1}, \ldots, A_{i}^{m_{i}}$.

Proof. The direction $\Leftarrow$ is trivial. For the converse, we merely need to show it for a single $A_{i}^{j}$ that we denote by $A$ for ease of notation. If for instance

$$
\lambda_{1} v_{1}+\lambda_{2} v_{2} \in F_{i}^{l}
$$

for some $\lambda_{j} \neq 0$ and $v_{j}$ in the $\zeta_{j}$-eigenspace of $A$ with $\zeta_{1} \neq \zeta_{2}$ then

$$
A\left(\lambda_{1} v_{1}+\lambda_{2} v_{2}\right)=\zeta_{1}\left(\lambda_{1} v_{1}+\lambda_{2} v_{2}\right)+\left(\zeta_{2}-\zeta_{1}\right) \lambda_{2} v_{2}
$$

Now as by assumption the left-hand side and the first term on the right-hand side belong to $F_{i}^{l}$, the same thing follows for the second term on the righthand side, and thus (as $\zeta_{2}-\zeta_{1} \neq 0$ ) for $\lambda_{2} v_{2}$ too, which in turn implies the same thing for $\lambda_{1} v_{1}$ as well. The same kind of argument applies for a vector with components in more than just two different eigenspaces.

A compatible parabolic structure at $p_{i}$ is the datum of a compatible quasiparabolic structure at $p_{i}$ and parabolic weights

$$
1>\alpha_{i}^{l_{i}-1}>\cdots>\alpha_{i}^{0} \geq 0 .
$$

2.2. Parabolic sheaves. It is convenient to recall the notion of an $\mathbf{R}$-parabolic sheaf on a complex manifold $X$ (or more generally, a non-singular projective variety over an algebraically closed field) with divisor $D_{\text {red }}$, a reduced effective Weil divisor on $X$ : this is a coherent sheaf $S$ with a decreasing filtration $S$. indexed by $\mathbf{R}$ so that for all $\alpha \in \mathbf{R}$

(1) there exists some $\varepsilon>0$ with $S_{\alpha-\varepsilon}=S_{\alpha}$;

(2) we have $S_{\alpha+1}=S_{\alpha} \otimes \mathcal{O}_{X}\left(-D_{\text {red }}\right)$;

(3) $S_{0}=S$.

Then we have the following result (Section 1, [26]):

Claim 2.2. The categories of $\mathbf{R}$-parabolic locally free sheaves on $X$ with divisor $D_{\text {red }}$ and of parabolic bundles on $X$ with divisor $D_{\text {red }}$ are isomorphic.

For convenience, let us spell out the correspondence in the case $X=$ $C$ a smooth projective curve. To a parabolic bundle $\mathcal{E}$ with filtration (4) associate the $\mathbf{R}$-parabolic sheaf $\mathcal{E}_{\bullet}$ defined as follows. Near the generic 
point $z \notin D_{\text {red }}$ for every $\alpha \in \mathbf{R}$ we let $\mathcal{E}_{\alpha}=\mathcal{E}$. For $i \in\{1, \ldots, n\}$ and $\alpha_{i}^{l-1}<\alpha \leq \alpha_{i}^{l}$ we define $\mathcal{E}_{\alpha}$ in a small neighborhood of $p_{i} \in D$ not containing any other $p_{i^{\prime}}$ as the kernel of the composition map

$$
\pi_{i}^{l}:\left.\mathcal{E} \stackrel{\text { eval }_{p_{i}}}{\longrightarrow} \mathcal{E}\right|_{p_{i}}=F_{i}^{0} \rightarrow F_{i}^{0} / F_{i}^{l} .
$$

For $\alpha \notin[0,1]$ we extend the definition by property (2) above.

Conversely, to an $\mathbf{R}$-parabolic sheaf we associate the vector bundle whose local sections are given by the sheaf $\mathcal{E}_{0}$, with filtration (4) defined as follows: for any vector $v \in \mathcal{E}_{\mid p_{i}}$ we let $v \in F_{i}^{l}$ if and only if any local section of $\mathcal{E}_{0}$ extending $v$ (i.e., whose specialization at $p_{i}$ is $v$ ) is actually a section of $\mathcal{E}_{\alpha_{i}^{l}}$.

These two constructions are clearly inverse to each other.

Based on this correspondence, it is natural to extend the defintion of an $\mathbf{R}$-parabolic sheaf to a more general setup, that we will use throughout the article. Let $X$ now stand for a (not necessarily smooth) complex curve and $\mathcal{L}$ denote a (not necessarily reduced) effective Cartier divisor on $X$.

Definition 2.3. An $\mathbf{R}$-parabolic sheaf over $X$ with parabolic divisor $\mathcal{L}$ is a decreasing family $S$. of coherent sheaves over $X$ indexed by $\mathbf{R}$ satisfying the left-continuity property (1) above, and moreover such that we have

$$
S_{\alpha+1}=S_{\alpha} \otimes \mathcal{L}^{\vee} \text {. }
$$

2.3. A ruled surface. Finally, let us introduce some notation concerning ruled surfaces. Let us denote by $Z$ the surface

$$
Z=\mathbf{P}_{C}\left(\mathcal{O}_{C} \oplus L^{\vee}\right)
$$

the fiberwise projectivisation of the total space of the line bundle $L^{\vee}$ dual to $L$. There exist a natural projection

$$
p: Z \rightarrow C,
$$

a relative hyperplane bundle $\mathcal{O}_{Z}(1)$ and canonical sections

$$
\xi \in H^{0}\left(Z, \mathcal{O}_{Z}(1)\right), \zeta \in H^{0}\left(Z, p^{*} L \otimes \mathcal{O}_{Z}(1)\right) .
$$

The divisors

$$
(\xi=0),(\zeta=0)
$$

are called the divisor at infinity and the 0-divisor respectively. The line bundle $\mathcal{O}_{Z}(1)$ is trivial on the complement of the divisor at infinity, and the same holds for the 0-divisor.

\section{THE CORRESPONDENCE IN THE LOGARITHMIC CASE}

In this section we will briefly outline the refined correspondence in the parabolic logarithmic (regular singular) case. The upshot is that it is possible to encode the compatible parabolic structure of the Higgs bundle in terms of a parabolic structure of the spectral sheaf. This is related to the 
treatment of [17] Proposition 2.2 except that we take a slightly different point of view as them: instead of partitioning the eigenvalues of the Higgs field into the graded pieces of a given filtration, we take the direct sum of the eigenspaces (the fibers of the spectral sheaf over the points lying above a parabolic point of $C$ ) and exhaust this space by an increasing filtration.

Let us first recall the extension by D. Schaub of the BNR-correspondence to the case of arbitrary spectral curves [21]. Fix an arbitrary element

$$
\vec{s}=\left\{s_{k}\right\}_{k=1}^{r} \in \oplus_{k=1}^{r} H^{0}\left(C, L^{k}\right)
$$

and let $\Sigma_{\vec{s}}$ be the corresponding spectral scheme of degree $r$ over $C$, i.e. $\Sigma_{\vec{s}}$ is the subscheme of $Z$ defined by the polynomial

$$
P_{\vec{s}}(\zeta, \xi)=\zeta^{r}+p^{*}\left(s_{1}\right) \zeta^{r-1} \xi+\cdots+p^{*}\left(s_{n}\right) \xi^{r} .
$$

For convenience, we sometimes denote $\Sigma_{\vec{s}}$ merely by $\Sigma$, when this may cause no confusion. Let $\mathcal{R}_{\Sigma}$ be the sheaf of rational functions on $\Sigma$ and let us be given a coherent sheaf of $\mathcal{O}_{\Sigma}$-modules $S$. Then $S$ is said to be torsion-free if the natural morphism

$$
S \rightarrow S \otimes_{\mathcal{O}_{\Sigma}} \mathcal{R}_{\Sigma}
$$

is injective. Let $S c h_{\mathbf{C}}$ and $S e t$ stand for the categories of schemes over $\mathbf{C}$ and of sets respectively. Let $\cong$ stand for the equivalence relation given by isomorphisms in the categories of sheaves and Higgs bundles. Consider the contravariant functors

- $F_{\vec{s}}: S c h_{\mathbf{C}} \rightarrow$ Set defined by

$F_{\vec{s}}(T)=\left\{S_{T}\right.$ a coherent $\mathcal{O}_{\Sigma_{\vec{s}} \times T}-$ module, flat over $T$ and inducing torsion-free rank $1 \mathcal{O}_{\Sigma_{\vec{s}} \times\{t\}}$ - modules on the geometric fibers of the projection $\left.\Sigma_{\vec{s}} \times T \rightarrow T\right\} / \cong$

- and $G_{\vec{s}}: S c h_{\mathbf{C}} \rightarrow$ Set defined by

$$
\begin{aligned}
G_{\vec{s}}(T)=\{ & \left(\mathcal{E}_{T}, \theta_{T}\right) \text { where } \mathcal{E}_{T} \text { is a locally free } \mathcal{O}_{C \times T}-\text { module of rank } r \\
& \text { and } \theta_{T}: \mathcal{E}_{T} \rightarrow \mathcal{E}_{T} \otimes p_{C}^{*} L \text { a morphism of } \mathcal{O}_{C \times T}-\text { modules } \\
& \text { with characteristic polynomial given by } \left.P_{\vec{s}}\right\} / \cong
\end{aligned}
$$

on objects, and associating the pull-back morphism on such objects to a morphism of schemes $T^{\prime} \rightarrow T$. With this notation, the functors $F_{\vec{s}}$ and $G_{\vec{s}}$ are proved to be isomorphic in Proposition 5.1 [21]. In the rest of this section, we upgrade this correspondence to a correspondence between parabolic torsion-free sheaves and logarithmic Higgs bundles endowed with a compatible parabolic structure.

Let $(\mathcal{E}, \theta)$ be a Higgs bundle on a smooth projective curve $C$ and let us assume that all the singularities of $\theta$ are logarithmic (i.e. $Q_{i}=0$ in (1)). 
Then for all $i, l$ the restriction of $\theta$ induces a map

$$
\mathcal{E}_{\alpha_{i}^{l}} \rightarrow \mathcal{E} \otimes L
$$

and since $\theta$ is assumed to be compatible with the parabolic structure, the composition

$$
\mathcal{E}_{\alpha_{i}^{l}} \rightarrow \mathcal{E} \otimes L \stackrel{\pi_{i}^{l} \otimes I d_{L}}{\longrightarrow} F_{i}^{0} /\left.F_{i}^{l} \otimes L\right|_{p_{i}}
$$

vanishes. We infer that the restriction of $\theta$ to $\mathcal{E}_{\alpha_{i}^{l}}$ factors through $\mathcal{E}_{\alpha_{i}^{l}} \otimes L$, and hence gives rise to maps

$$
\theta_{\alpha}: \mathcal{E}_{\alpha} \rightarrow \mathcal{E}_{\alpha} \otimes L
$$

for every $\alpha$. To emphasize that we are dealing with the logarithmic case set

$$
Z_{\text {red }}=\mathbf{P}_{C}\left(\mathcal{O}_{C} \oplus K_{C}\left(D_{\text {red }}\right)^{\vee}\right)
$$

and for ease of notation let us denote by $p, \zeta, \xi$ the corresponding projection from $Z_{\text {red }}$ to $C$ and canonical sections. It follows from what we have said above that we can define the spectral sheaves corresponding to the subsheaves $\mathcal{E}_{\alpha}$ :

$$
S_{\mathcal{E}_{\alpha}}=\operatorname{coker}\left(p^{*} \mathcal{E}_{\alpha} \stackrel{\xi \otimes p^{*} \theta+\zeta}{\longrightarrow} p^{*}\left(\mathcal{E}_{\alpha} \otimes K_{C}\left(D_{\text {red }}\right)\right) \otimes \mathcal{O}_{Z_{\text {red }}}(1)\right) .
$$

This is a coherent sheaf whose support is denoted

$$
\Sigma=\left(\operatorname{det}\left(\xi \otimes p^{*} \theta+\zeta\right)\right)
$$

and called spectral curve. (In principle, $\Sigma$ could depend on $\alpha$, but the proof of the proposition below shows in particular that this is not the case.)

Proposition 3.1. (1) If $(\mathcal{E}, \theta)$ is a logarithmic Higgs bundle with compatible parabolic structure at $D_{\mathrm{red}}$ on a curve $C$ then the sheaves $S_{\mathcal{E}_{\alpha}}$ are pure of dimension 1 and form an $\mathbf{R}$-parabolic sheaf on $Z_{\text {red }}$ with divisor $p^{-1}\left(D_{\text {red }}\right)$.

(2) Conversely, given any $\mathbf{R}$-parabolic sheaf $S_{\alpha}$ with divisor $p^{-1}\left(D_{\text {red }}\right)$ and sheaves pure of dimension 1 on $Z_{\mathrm{red}}$ with support disjoint from $\xi=0$, then the sheaves $\mathcal{E}_{\alpha}=p_{\star} S_{\alpha} \otimes K_{C}\left(D_{\text {red }}\right)^{\vee}$ form an $\mathbf{R}$-parabolic bundle on $C$ and $\theta$ given by

$$
\theta=p_{\star}\left(-\zeta \cdot: S_{\alpha} \otimes K_{C}\left(D_{\text {red }}\right)^{\vee} \rightarrow S_{\alpha}\right)
$$

defines a Higgs field with regular singularities and compatible with the parabolic structure.

The two constructions are quasi-inverse to each other.

Proof. By [4], we have

$$
p_{\star} S_{\mathcal{E}_{\alpha}}=\mathcal{E}_{\alpha} \otimes L
$$


If the schematic support of $S_{\mathcal{E}_{\alpha}}$ had a 0-dimensional embedded component, then the same would hold for $\mathcal{E}_{\alpha} \otimes L$; this however is impossible as $\mathcal{E}_{\alpha}$ is locally free, in particular torsion-free.

For any $\alpha<\beta$ the quotient $\mathcal{E}_{\alpha} / \mathcal{E}_{\beta}$ is a skyscaper sheaf supported at $D_{\text {red }}$; let us denote this sheaf by $\mathbf{C}^{\alpha, \beta}$. Now since $p^{*} \mathbf{C}^{\alpha, \beta}$ is pure of dimension 1 supported on $p^{-1}\left(D_{\text {red }}\right)$ and the map $p^{*} \theta+\zeta$ is non-zero on this sheaf (unless it is itself the 0-sheaf), its kernel must vanish and we have the diagram

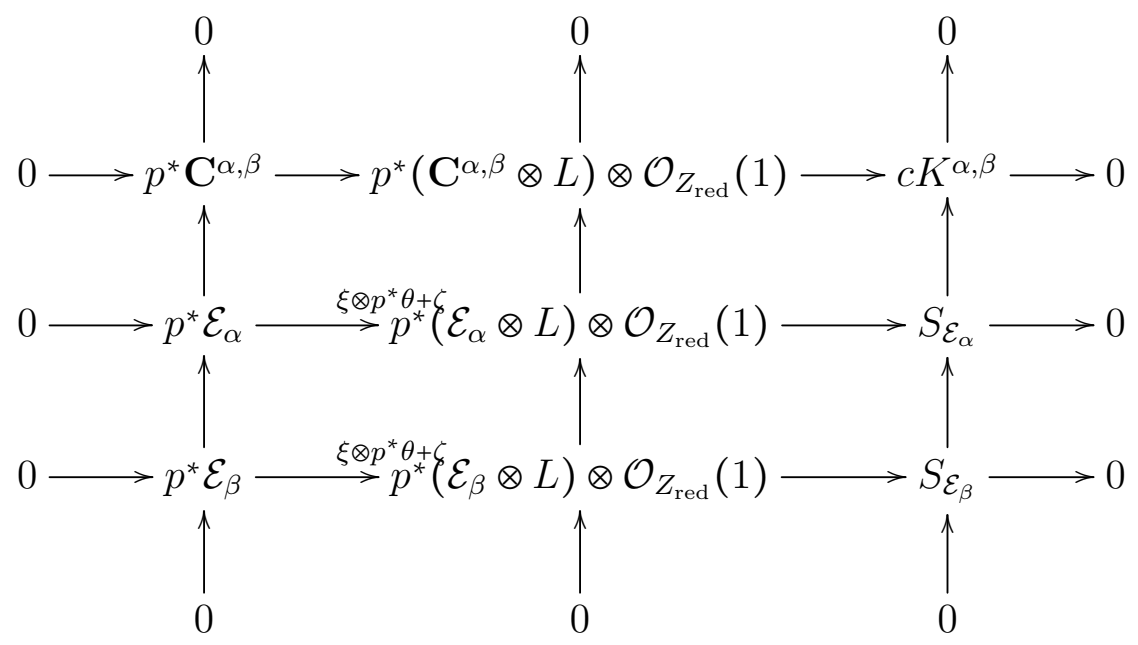

which defines the cokernel sheaf $c K^{\alpha, \beta}$. The top row shows that $c K^{\alpha, \beta}$ is a torsion sheaf supported in dimension 0 over

$$
\Sigma \cap p^{-1}\left(D_{\text {red }}\right)
$$

so $S_{\mathcal{E}_{\alpha}}$ is an upper elementary modification of $S_{\mathcal{E}_{\beta}}$ at these points. This shows that the sequence of sheaves $S_{\mathcal{E}_{\bullet}}$ is decreasing. Now for any $\alpha$ there exists $\varepsilon>0$ such that we have $\mathcal{E}_{\alpha-\varepsilon}=\mathcal{E}_{\alpha}$; it then follows that $S_{\mathcal{E}_{\alpha-\varepsilon}}=S_{\mathcal{E}_{\alpha}}$ too. Finally, as $\mathcal{E}_{\alpha+1}=\mathcal{E}_{\alpha}\left(-\left(z_{0}+\cdots+z_{n}\right)\right)$ the projection formula

$$
p^{*}\left(\mathcal{O}_{C}\left(D_{\text {red }}\right)\right)=\mathcal{O}_{Z_{\text {red }}}\left(p^{-1}\left(D_{\text {red }}\right)\right),
$$

implies

$$
S_{\mathcal{E}_{\alpha+1}}=S_{\mathcal{E}_{\alpha}}\left(-p^{-1}\left(D_{\text {red }}\right)\right) .
$$

For the converse direction, all the sheaves $\mathcal{E}_{\alpha}$ are locally free as torsionfree sheaves on smooth curves are locally free. As multiplication by $(-\zeta)$ preserves $S_{\alpha}$ it follows that $\theta$ preserves $\mathcal{E}_{\alpha}$, which in turn means that $\theta$ is compatible with the parabolic filtration. Finally, the fact that $\mathcal{E}_{\alpha}$ is $\mathbf{R}$ parabolic follows again from (10).

The fact that the two constructions are inverse to each other is straightforward [4]: a $p_{*} \mathcal{O}_{\Sigma}$-module structure on $\mathcal{E}$ is the same thing as an $\mathcal{O}_{C}$-linear 
endomorphism $\theta$, and over the locus where the eigenvalues of $\theta$ are all distinct the $p_{*} \mathcal{O}_{\Sigma}$-module structure simply gives the decomposition of $\mathcal{E}$ into its eigenspaces for $\theta$.

Example 1. Let us illustrate how this result works when $\Sigma$ is ramified over $C$. Specifically, let us consider $C=$ Spec $\mathbf{C}[z]$; we will identify coherent modules over $\mathrm{C}[z]$ with their associated coherent sheaves on $C$. We let the divisor $D_{\text {red }}$ be given by $(z) \triangleleft \mathrm{C}[z]$ and assume $\mathcal{E}_{0}$ is a free $\mathrm{C}[z]$-module of rank 2

$$
\mathcal{E}_{0}=\mathbf{C}[z] \mathbf{e}_{0} \oplus \mathbf{C}[z] \mathbf{e}_{1}
$$

with

$$
\theta\left(\mathbf{e}_{0}\right)=\mathbf{e}_{1} \frac{d z}{z}, \quad \theta\left(\mathbf{e}_{1}\right)=\mathbf{e}_{0} d z .
$$

Then, trivializing $K_{C}(D)$ by the section $d z / z$, we get $\Sigma=\operatorname{Spec} \mathrm{C}[z, \zeta] /(z-$ $\left.\zeta^{2}\right)$. This ring is isomorphic to $\mathrm{C}[\zeta]$ and the map $p$ is then $\zeta \mapsto \zeta^{2}$. Furthermore, $S_{\mathcal{E}_{0}}$ is isomorphic to (the sheaf induced by) a free rank 1 module over $\mathrm{C}[\zeta]$. Identifying this module to $\mathrm{C}[\zeta]$ itself, the push-forward morphism is

$$
p_{\star}(1)=\mathbf{e}_{0}, \quad p_{\star}(\zeta)=\mathbf{e}_{1} .
$$

The Galois-group of $p: \Sigma \rightarrow C$ is $\mathbf{Z} /(2)$, and the line subbundles spanned by the two trivializing sections $\mathbf{e}_{0}, \mathbf{e}_{1}$ are naturally isomorphic to the equivariant sections of this action corresponding to the trivial multiplicative character and the unique non-trivial multiplicative character acting by $\zeta \mapsto$ $-\zeta$, respectively. The pull-back of the Cartier divisor $(z)$ to $\Sigma$ is $\left(\zeta^{2}\right) \triangleleft$ $\mathrm{C}[\zeta]$, corresponding to a non-reduced divisor. Now, for a compatible parabolic structure there are two possibilities.

First, we may consider the trivial filtration

$$
\{0\} \subset \mathbf{C e}_{0} \oplus \mathbf{C e}_{1}
$$

associated to a weight $0 \leq \alpha_{0}<1$. For $\alpha \in[0,1)$ the associated $\mathbf{R}$-parabolic sheaf is given by

$$
\mathcal{E}_{\alpha}=\mathcal{E}_{0} \quad \text { for } \alpha \leq \alpha_{0}
$$

and

$$
\mathcal{E}_{\alpha}=\mathcal{E}_{0} \otimes_{\mathbf{C}[z]} z \mathbf{C}[z] \text { for } \alpha_{0}<\alpha .
$$

The $\mathrm{C}[\zeta]$-modules associated to these $\mathrm{C}[z]$-modules are

$$
S_{\mathcal{E}_{\alpha}}=\mathbf{C}[\zeta] \text { for } \alpha \leq \alpha_{0}
$$

and

$$
S_{\mathcal{E}_{\alpha}}=\zeta^{2} \mathbf{C}[\zeta] \text { for } \alpha_{0}<\alpha .
$$

We see that these modules satisfy the requirements of Definition 2.3 for the reduced divisor $\left(\zeta^{2}\right) \triangleleft \mathbf{C}[\zeta]$, with just one jump $\alpha_{0}$ for values of $\alpha$ between 0 and 1 . 
As the image of $\operatorname{res}_{z=0} \theta$ is spanned by $\mathbf{e}_{1}$, the only other compatible filtration is

$$
\{0\} \subset \mathbf{C e}_{1} \subset \mathbf{C e}_{0} \oplus \mathbf{C e}_{1},
$$

with the two inclusions corresponding to some weights $0 \leq \alpha_{-}<\alpha_{+}<1$. The corresponding $\mathbf{R}$-parabolic bundle is characterized by

$$
\begin{array}{rlrl}
\mathcal{E}_{\alpha} & =\mathcal{E}_{0} & & \text { for } 0 \leq \alpha \leq \alpha_{-} \\
& =z \mathbf{C}[z] \mathbf{e}_{0} \oplus \mathbf{C}[z] \mathbf{e}_{1} & & \text { for } \alpha_{-}<\alpha \leq \alpha_{+} \\
& =\mathcal{E}_{0} \otimes_{\mathbf{C}[z]} z \mathbf{C}[z] & \text { for } \alpha_{+}<\alpha<1,
\end{array}
$$

extended using the periodicity property (2) above. The corresponding $\mathbf{R}$ parabolic sheaf on $\Sigma i$ s

$$
\begin{array}{rlr}
S_{\mathcal{E}_{\alpha}} & =\mathbf{C}[\zeta] & \text { for } \alpha \leq \alpha_{-} \\
& =\zeta \mathbf{C}[\zeta] & \text { for } \alpha_{-}<\alpha \leq \alpha_{+} \\
& =\zeta^{2} \mathbf{C}[\zeta] & \text { for } \alpha_{+}<\alpha .
\end{array}
$$

This again satisfies Definition 2.3 for the reduced divisor $\left(\zeta^{2}\right)$, this time with two jumps $0 \leq \alpha_{-}<\alpha_{+}<1$.

Example 2. Let us study the simplest situation in which the map $p: \Sigma \rightarrow C$

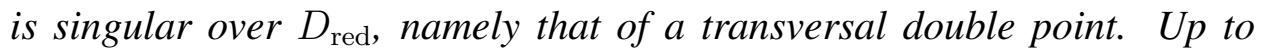
isomorphism there are two kinds of torsion-free sheaves of $\mathcal{O}_{\Sigma}$-modules of rank 1: line bundles and sheaves that are direct images of line bundles on the normalization $\tilde{\Sigma}$ of $\Sigma$. As $\tilde{\Sigma}$ is smooth over $C$, the latter case can easily be understood, hence we restrict ourselves to the case of a line bundle over $\Sigma$. The analysis is quite similar to Example 1, so we will not give all the details. Let us again set $C=\operatorname{Spec} \mathbf{C}[z], D_{\text {red }}=(z)$ and

$$
\mathcal{E}_{0}=\mathbf{C}[z] \mathbf{e}_{0} \oplus \mathbf{C}[z] \mathbf{e}_{1} .
$$

Let the logarithmic Higgs field be given by

$$
\theta\left(\mathbf{e}_{0}\right)=\mathbf{e}_{1} \frac{d z}{z}, \quad \theta\left(\mathbf{e}_{1}\right)=\mathbf{e}_{0} z d z .
$$

Trivializing $K_{C}(D)$ by $d z / z$ we now get

$$
\Sigma=\operatorname{Spec}\left(\mathbf{C}[z, \zeta] /\left(z^{2}-\zeta^{2}\right)\right)
$$

and $S_{\mathcal{E}_{0}}=\mathcal{O}_{\Sigma}$. We again have

$$
p_{\star}(1)=\mathbf{e}_{0}, \quad p_{\star}(\zeta)=\mathbf{e}_{1},
$$

and the same two possible types of compatible parabolic structures can occur as in Example 1.

In the case of a trivial filtration, just as in the ramified case we get

$$
S_{\mathcal{E}_{\alpha}}=\mathcal{O}_{\Sigma} \quad \text { for } \alpha \leq \alpha_{0}
$$


and

$$
S_{\mathcal{E}_{\alpha}}=z \mathcal{O}_{\Sigma} \text { for } \alpha_{0}<\alpha .
$$

Indeed, the vector space $\mathcal{O}_{\Sigma} /(z)$ is spanned by the elements 1 and $\zeta$ of $\mathcal{O}_{\Sigma}$, and these correspond to the vectors $\mathbf{e}_{0}, \mathbf{e}_{1}$ respectively.

In the full flag case (11), the formulae for $\mathcal{E}_{\alpha}$ are the same as in the ramified situation, and for the associated $\mathbf{R}$-parabolic sheaf $S_{\mathcal{E}_{\alpha}}$ just as above we get

$$
S_{\mathcal{E}_{\alpha}}=\mathcal{O}_{\Sigma} \quad \text { for } \alpha \leq \alpha_{-}
$$

and

$$
S_{\mathcal{E}_{\alpha}}=z \mathcal{O}_{\Sigma} \text { for } \alpha_{+}<\alpha .
$$

For $\alpha_{-}<\alpha \leq \alpha_{+}$the module $S_{\mathcal{E}_{\alpha}}$ is given as the subalgebra of $\mathcal{O}_{\Sigma}$ generated by $z$ and $\zeta$. Clearly, the quotient of $\mathcal{O}_{\Sigma}$ with this algebra is spanned by $1 \in \mathcal{O}_{\Sigma}$, whose image by $p_{\star}$ is $\mathbf{e}_{0}$. As $\mathbf{e}_{0}$ is a generator of

$$
\operatorname{ker}\left(\mathbf{C e}_{0} \oplus \mathbf{C} \mathbf{e}_{1} \rightarrow \mathbf{C e}_{1}\right),
$$

the quasi-parabolic filtration associated to $p_{*} S_{\mathcal{E}_{\alpha}}$ for these values of $\alpha$ is indeed (11).

Proposition 3.2. There exists an isomorphism of categories between the functors

- $F_{\vec{s}}^{\text {par }}:$ Sch $\mathbf{C} \rightarrow$ Set defined by

$F_{\vec{s}}^{\text {par }}(T)=\left\{S_{T}\right.$ a coherent $\mathcal{O}_{\Sigma_{\vec{s} \times T}}-$ module, flat over $T$

and inducing torsion-free rank 1 parabolic $\mathcal{O}_{\Sigma_{\vec{s} \times\{t\}}}$ - modules

on the geometric fibers of the projection $\Sigma_{\vec{s}} \times T \rightarrow T$

with parabolic divisor $\left.\left(p_{\Sigma} \circ p\right)^{*}\left(D_{\text {red }}\right)\right\} / \cong$

- and $G_{\vec{s}}^{p a r}: S c h_{\mathbf{C}} \rightarrow$ Set defined by

$G_{\vec{s}}^{\text {par }}(T)=\left\{\left(\mathcal{E}_{T}, \theta_{T}\right)\right.$ where $\mathcal{E}_{T}$ is a locally free parabolic $\mathcal{O}_{C \times T}-$ module of rank $r$ with parabolic divisor $p_{C}^{*}\left(D_{\text {red }}\right)$

and $\theta_{T}: \mathcal{E}_{T} \rightarrow \mathcal{E}_{T} \otimes p_{C}^{*} L$ a morphism of $\mathcal{O}_{C \times T}-$ modules

with characteristic polynomial given by $P_{\vec{s}}$

and action on the geometric fibers of the projection $C \times T \rightarrow T$

compatible with the parabolic structure of $\left.\mathcal{E}_{t}\right\} / \cong$

Proof. Follows from the previous proposition along the same lines as Proposition 5.1 [21] from Proposition 2.1 [21]. 


\section{THE CORRESPONDENCE IN THE IRREGULAR CASE}

Proposition 3.2 gives a satisfactory result at the regular singularities; however, at the irregular singularities the sheaves must possess a finer structure in order for such a correspondence to hold. This structure may be conveniently expressed using the technique of proper transform of $\mathbf{R}$-parabolic sheaves defined in Section 5 of [1]. Let us now describe this construction. To fix our ideas, let us first treat the case $m_{i}=1$, and set $A_{i}=A_{i}^{1}$. Fix a local chart $z_{i}$ vanishing at $p_{i}$; this then gives rise to a local trivialization

$$
\lambda_{i}=z_{i}^{-2} \mathrm{~d} z_{i}
$$

of the line bundle (2). The assumption $m_{i}=1$ means that $\theta$ has local form

$$
\theta=\left(-A_{i}+z_{i} \Lambda_{i}+\cdots\right) \lambda_{i}
$$

where the dots stand for higher order terms in $z_{i}$. Let us introduce the local holomorphic function $\zeta_{i}$ on $Z \backslash(\xi)$ by

$$
\zeta=\zeta_{i} p^{*} \lambda_{i}
$$

as the left hand side is a section of $p^{*} L$ and $p^{\star} \lambda_{i}$ is a local section of $L, \zeta_{i}$ is then a local function. In what follows, we will drop $p^{*}$ from the notation. Let us denote the distinct eigenvalues of $-A_{i}$ by $\zeta_{i, 1}, \ldots, \zeta_{i, L_{i}}$, each repeated with a certain multiplicity $d_{i, 1}, \ldots, d_{i, L_{i}}$. It is known that the residue $\Lambda_{i}$ may be assumed up to a gauge transformation to be block diagonal with respect to the eigenspace-decomposition of $A_{i}$ (actually, the same thing holds for any prescribed number of the lower order terms). Let us denote the block of $\Lambda_{i}$ corresponding to the eigenvalue $\zeta_{i, l}$ of $-A_{i}$ by $\Lambda_{i, l}$. The spectral curve $\Sigma$ passes through the points $\left(p_{i}, \zeta_{i, 1}\right), \ldots,\left(p_{i}, \zeta_{i, L_{i}}\right)$ (where in the second coordinate $\zeta_{i, l}$ means the point $\left(\zeta_{i, l}: 1\right)$ in the projective chart of the fiber $\left.\mathcal{E}\right|_{p_{i}}$ given by the sections $\zeta, \xi$ ). Let us consider the formal splitting of $\mathcal{E}$ into the generalised eigenspaces for $\theta$ near $p_{i}$ :

$$
\mathcal{E}=\mathcal{E}^{i, 1} \oplus \cdots \oplus \mathcal{E}^{i, r}
$$

this splitting holds over the field of formal Puiseux-series in $z_{i}$. It can be shown (c.f. Claim 4.27 [23]) using Rouché's root counting formula that the eigenvalues $\zeta_{i, j}$ of $\theta$ are of the form

$$
\left(\frac{\zeta_{i, j}}{z_{i}^{2}}+O\left(z_{i}^{-2+\frac{1}{r}}\right)\right) \mathrm{d} z_{i}
$$

where for $1 \leq j \leq r$ we denote by $\zeta_{i, j}$ the $j$ 'th eigenvalue of $-A_{i}$ in the compatible basis of (13). Let us gather the generalised eigenspaces appearing in (15) corresponding to equal values of $\zeta_{i, j}$ to define a coarser decomposition

$$
\mathcal{E}=\tilde{\mathcal{E}}^{i, 1} \oplus \cdots \oplus \tilde{\mathcal{E}}^{i, L_{i}}
$$


for example,

$$
\tilde{\mathcal{E}}^{i, 1}=\bigoplus_{j} \mathcal{E}^{i, j}
$$

the summation ranging for all values of $j$ such that $\zeta_{i, j}$ is equal to a fixed complex number, etc.

Claim 4.1. The decomposition (17) is defined over the field $\mathbf{C}\left\{z_{i}\right\}\left[z_{i}^{-1}\right]$ of convergent Laurent series in the variable $z_{i}$, and the summands are locally free $\mathbf{C}\left\{z_{i}\right\}$-modules. Furthermore, it is preserved by $\theta$.

Proof. Let $L$ be the splitting field of the characteristic polynomial

$$
\chi_{\theta\left(\partial_{z_{i}}\right)}\left(\zeta, z_{i}\right)=\operatorname{det}\left(\theta\left(\partial_{z_{i}}\right)+\zeta\right)
$$

of $\theta\left(\partial_{z_{i}}\right)$ over the ring of formal power series $\mathbf{C}\left[\left[z_{i}\right]\right]$. Then the action of $\operatorname{Gal}\left(L \mid \mathbf{C}\left[\left[z_{i}\right]\right]\right)$ maps a generalised eigenvector with Puiseux-expansion having highest-order term $\zeta_{i, j}$ to a generalised eigenvector with equal highestorder term. Therefore, the direct sum of such generalised eigenspaces is $\operatorname{Gal}\left(L \mid \mathbf{C}\left[\left[z_{i}\right]\right]\right)$-invariant. On the other hand, the solution curve of $\chi_{\theta\left(\partial_{z_{i}}\right)}$ is the graph of a multi-valued analytic function in $z_{i}$, hence the generalised eigenvectors are given by convergent Puiseux-series. As $\mathcal{E}$ is torsion-free, it may only have torsion-free submodules. This proves the first two statements.

As for the third statement, notice that as the factors $\mathcal{E}^{i, l}$ are direct sums of generalised $\theta$-eigenspaces, they are preserved by $\theta$ over the generic point. So for any $l \neq l^{\prime}$ the map

$$
\mathcal{E}^{i, l} \rightarrow \mathcal{E}^{i, l^{\prime}}
$$

induced by $\theta$ is generically 0 . We conclude by the second statement.

To simplify notation, from now on we will write $\mathcal{E}^{i, l}$ for $\tilde{\mathcal{E}}^{i, l}$. Let $\theta^{i, l}$ stand for the restriction of $\theta$ to $\mathcal{E}^{i, l}$. The claim implies that we have a local direct sum decomposition

$$
(\mathcal{E}, \theta)=\left(\mathcal{E}^{i, 1}, \theta^{i, 1}\right) \oplus \cdots \oplus\left(\mathcal{E}^{i, L_{i}}, \theta^{i, L_{i}}\right)
$$

with $\left.\mathcal{E}^{i, l}\right|_{p_{i}}$ equal to the $\zeta_{i, l}$-eigenspace of $A$. Furthermore, by Claim 2.1, the above splitting is also compatible with the parabolic structure in the sense that for any $\alpha$ we also have

$$
\mathcal{E}_{\alpha}=\mathcal{E}_{\alpha}^{i, 1} \oplus \cdots \oplus \mathcal{E}_{\alpha}^{i, L_{i}}
$$

where

$$
\mathcal{E}_{\alpha}^{i, l}=\mathcal{E}_{\alpha} \cap \mathcal{E}^{i, l}
$$

Define the affine curves $\Sigma^{i, l}$ by the vanishing of the sections

$$
\operatorname{det}\left(\zeta \mathbf{I}_{\mathcal{E}^{i, l}}+\xi \theta^{i, l}\right)
$$


where $\mathrm{I}_{\mathcal{E}^{i, l}}$ stands for the identity transformation of $\mathcal{E}^{i, l}$. Let us now blow up $Z$ at the points $\left(p_{i}, \zeta_{i, l}\right)$ for $l \in\left\{1, \ldots, L_{i}\right\}$ :

$$
\sigma_{i}: Z^{i} \longrightarrow Z \text {. }
$$

The exceptional divisor of $\sigma_{i}$ corresponding to $\left(p_{i}, \zeta_{i, l}\right)$ is denoted $E_{i, l}$. Specifically, introduce homogeneous coordinates

$$
\left(z_{i, l}^{\prime}: \zeta_{i, l}^{\prime}\right) \in E_{i, l}=\mathbf{P}^{1}
$$

and let $U_{i, l}$ denote a neighborhood of $\left(p_{i}, \zeta_{i, l}\right)$ containing no other point $\left(p_{i^{\prime}}, \zeta_{i^{\prime}, l^{\prime}}\right)$ for $i^{\prime} \neq i, l^{\prime} \neq l$. Then the preimage by $\sigma_{i}$ of $U_{i, l}$ is given by

$$
\sigma_{i}^{-1}\left(U_{i, l}\right)=\left\{z_{i} \zeta_{i, l}^{\prime}=z_{i, l}^{\prime}\left(\zeta_{i}-\zeta_{i, l}\right)\right\} \subset U_{i, l} \times \mathbf{P}^{1} .
$$

Let us denote by

$$
U_{i, l}^{\prime} \subset \sigma_{i}^{-1}\left(U_{i, l}\right)
$$

the affine subset defined by $z_{i, l}^{\prime} \neq 0$. On $U_{i, l}^{\prime}$ we then have

$$
\zeta_{i}-\zeta_{i, l}=z_{i} \frac{\zeta_{i, l}^{\prime}}{z_{i, l}^{\prime}}
$$

in particular, on $U_{i, l}^{\prime}$ the equality $z_{i}=0$ implies $\zeta_{i}=\zeta_{i, l}$. Here $\zeta_{i, l}^{\prime}, z_{i, l}^{\prime}$ are to be understood as sections of $\mathcal{O}_{Z^{i}}\left(-E_{i, l}\right)$, since

$$
\left.\mathcal{O}_{Z^{i}}\left(-E_{i, l}\right)\right|_{E_{i, l}} \cong \mathcal{O}_{E_{i, l}}(1) \text {. }
$$

The equation of the total transform $\sigma_{i}^{-1}(\Sigma)$ of the spectral curve in the above affine chart therefore writes

$$
\operatorname{det}\left(\zeta \mathbf{I}_{\mathcal{E}^{i, l}}-\theta^{i, l}\right)=\operatorname{det}\left(z_{i} \frac{\zeta_{i, l}^{\prime}}{z_{i, l}^{\prime}} \lambda_{i} \mathbf{I}_{\mathcal{E}^{i, l}}-\tilde{\theta}^{i, l}\right)
$$

with

$$
\tilde{\theta}^{i, l}=\theta^{i, l}-\zeta_{i, l} \lambda_{i} \mathrm{I}_{\mathcal{E}^{i, l}}=\left(B_{i, l}+O\left(z_{i}\right)\right) \frac{\mathrm{d} z_{i}}{z_{i}}=\left(B_{i, l}+O\left(z_{i}\right)\right) z_{i} \lambda_{i}
$$

as $z_{i} \rightarrow 0$. We now resolve the quotient in the determinant above by writing

$$
\operatorname{det}\left(\zeta \mathbf{I}_{\mathcal{E}^{i, l}}-\theta^{i, l}\right)=\left(\frac{1}{z_{i, l}^{\prime}}\right)^{d_{i, l}} \operatorname{det}\left(z_{i} \zeta_{i, l}^{\prime} \lambda_{i} \mathbf{I}_{\mathcal{E}^{i, l}}-z_{i, l}^{\prime} \tilde{\theta}^{i, l}\right)
$$

This is therefore the defining relation of the total transform $\sigma_{i}^{-1}(\Sigma)$; observe now that if

$$
z_{i}=0=\zeta_{i}-\zeta_{i, l}
$$

then the matrix in the argument of the right hand side of (23) vanishes for any $\left(z_{i, l}^{\prime}: \zeta_{i, l}^{\prime}\right)$. Said differently, $E_{i, l}$ is a component of multiplicity $d_{i, l}$ in 
$\sigma_{i}^{-1}(\Sigma)$. The proper transform $\Sigma^{i}$ of $\Sigma$ with respect to $\sigma_{i}$ is therefore given in the chart $U_{i, l}^{\prime}$ by the formula

$\operatorname{det}\left(\zeta_{i, l}^{\prime} \lambda_{i} \mathrm{I}_{\mathcal{E}^{i, l}}-z_{i, l}^{\prime} z_{i}^{-1} \tilde{\theta}^{i, l}\right) \in H^{0}\left(U_{i, l}^{\prime},\left(p \circ \sigma_{i}\right)^{*}\left(L \otimes \mathcal{E} n d\left(\mathcal{E}^{i, l}\right)\right) \otimes \mathcal{O}_{Z^{i}}\left(-E_{i, l}\right)\right)$

(compare with (6)). Notice that here

$$
z_{i}^{-1} \tilde{\theta}^{i, l}=\left(B_{i, l}+O\left(z_{i}\right)\right) \lambda_{i}
$$

is a regular section of $\left(p \circ \sigma_{i}\right)^{\star}\left(L \otimes \mathcal{E} n d\left(\mathcal{E}^{i, l}\right)\right)$. In particular, we see that (24) has precisely the same form as the characteristic equation (8) of a logarithmic Higgs field with residue $B_{i, l}$ with respect to the auxiliary variable $\left(\zeta_{i, l}^{\prime} \lambda_{i}: z_{i, l}^{\prime}\right)$ instead of $(\zeta: \xi)$. Just as in the regular case, from the assumption that $B_{i, l}$ preserves the parabolic filtration we deduce that $z_{i}^{-1} \tilde{\theta}^{i, l}$ restricts to maps

$$
z_{i}^{-1} \tilde{\theta}_{\alpha}^{i, l}:\left(p \circ \sigma_{i}\right)^{*} \mathcal{E}_{\alpha}^{i, l} \rightarrow\left(p \circ \sigma_{i}\right)^{*}\left(\mathcal{E}_{\alpha}^{i, l} \otimes L\right)
$$

of locally free sheaves over $U_{i, l}^{\prime}$; or equivalently, maps

$$
z_{i}^{-1} \tilde{\theta}_{\alpha}^{i, l}:\left(p \circ \sigma_{i}\right)^{*}\left(\mathcal{E}_{\alpha}^{i, l} \otimes L^{\vee}\right) \rightarrow\left(p \circ \sigma_{i}\right)^{*} \mathcal{E}_{\alpha}^{i, l} .
$$

Now the proper transform $\Sigma^{i}$ given by equation (24) has an obvious refinement in terms of the spectral sheaves $S_{\mathcal{E}_{\alpha}}$ too. Namely, analogously to (7) we may define coherent sheaves on $Z^{i}$ by the formulae

$$
S_{\mathcal{E}_{\alpha}}^{i}=\operatorname{coker}\left(\zeta_{i, l}^{\prime} \lambda_{i} \mathrm{I}_{\left(\mathcal{E}_{\alpha}^{i, l} \otimes L^{\vee}\right)}-z_{i, l}^{\prime} z_{i}^{-1} \tilde{\theta}_{\alpha}^{i, l}\right)
$$

locally on the affine charts $U_{i, l}^{\prime}$ and as $S_{\mathcal{E}_{\alpha}}$ away from these charts. The support of $S_{\mathcal{E}_{\alpha}}^{i}$ is then obviously equal to $\Sigma^{i}$.

Claim 4.2. Let $z_{i} \in U \subset C$ be an open set such that $z_{i^{\prime}} \notin U$ for $i^{\prime} \neq i$. Then on the open subset $\left(p \circ \sigma_{i}\right)^{-1} U \subset Z^{i}$ we have

$$
\left(p \circ \sigma_{i}\right)_{\star} S_{\mathcal{E}_{\alpha}}^{i}=\mathcal{E}_{\alpha} .
$$

The sheaves $S_{\mathcal{E}_{\bullet}}^{i}$ are pure and define an $\mathbf{R}$-parabolic sheaf with divisor

$$
\left(p \circ \sigma_{i}\right)^{-1}\left(p_{i}\right)
$$

Proof. Let us first show purity: this is a simple consequence of the AuslanderBuchsbaum formula [14] pp. 4-5: using that $Z^{i}$ is regular of dimension 2 at any point $x \in \Sigma^{i}$ and the projective dimension of $\left(S_{\mathcal{E}_{\alpha}}^{i}\right)_{x}$ is by definition equal to 1 we get that $\operatorname{depth}\left(\left(S_{\mathcal{E}_{\alpha}}^{i}\right)_{x}\right)=1$, so its schematic support does not have a 0-dimensional embedded component.

By definition for any $V \subseteq U$ we have

$$
\left(p \circ \sigma_{i}\right)_{\star} S_{\mathcal{E}_{\alpha}}^{i}(V)=S_{\mathcal{E}_{\alpha}}^{i}\left(\left(p \circ \sigma_{i}\right)^{-1}(V)\right) .
$$


As the support of $S_{\mathcal{E}_{\alpha}}^{i}$ is contained in the disjoint union of the open sets (21) for $l \in\left\{1, \ldots, L_{i}\right\}$, the right-hand side is a direct sum of the Abelian groups

$$
S_{\mathcal{E}_{\alpha}}^{i}\left(\left(p \circ \sigma_{i}\right)^{-1}(V) \cap U_{i, l}^{\prime}\right) .
$$

This latter is in turn obtained by the sheafification of the pre-sheaf

$$
\left(\left(p \circ \sigma_{i}\right)^{*} \mathcal{E}_{\alpha}^{i, l} / \operatorname{im}\left(\zeta_{i, l}^{\prime} \lambda_{i} \mathrm{I}_{\left(\mathcal{E}_{\alpha}^{i, l} \otimes L^{\vee}\right)}-z_{i, l}^{\prime} z_{i}^{-1} \tilde{\theta}_{\alpha}^{i, l}\right)\right)\left(\left(p \circ \sigma_{i}\right)^{-1}(V) \cap U_{i, l}^{\prime}\right) .
$$

Mapping a section of $\mathcal{E}_{\alpha}^{i, l}$ on $V$ to the class of its pull-back thus gives us a canonical epimorphism of sheaves of vector-spaces

$$
\iota: \oplus_{l=1}^{L_{i}} \mathcal{E}_{\alpha}^{i, l} \rightarrow\left(p \circ \sigma_{i}\right)_{\star} S_{\mathcal{E}_{\alpha}}^{i} .
$$

We now show that it is a monomorphism. Notice that both the source and target of $\iota$ are torsion-free sheaves on $C$ : for $\mathcal{E}_{\alpha}^{i, l}$ this holds as it is a subsheaf of the locally free sheaf $\mathcal{E}$, and for $\left(p \circ \sigma_{i}\right)_{*} S_{\mathcal{E}_{\alpha}}^{i}$ it follows from purity of $S_{\mathcal{E}_{\alpha}}^{i}$ since the support $\Sigma^{i}$ of this latter sheaf is finite over $C$. Therefore, it is sufficient to show that $\iota$ is generically a monomorphism. The support of $S_{\mathcal{E}_{\alpha}}^{i}$ is equal to $\Sigma^{i} \cap\left(p \circ \sigma_{i}\right)^{-1}(U)$; in particular by the assumption that $\Sigma^{i}$ is reduced, it is $r$ to 1 over $U$. On the open subset $U^{0} \subseteq U$ over which the $r$ branches of $\Sigma^{i}$ are distinct the map $\iota$ corresponds to writing sections of $\mathcal{E}_{\alpha}^{i, l}$ as $\mathcal{O}_{U}$-linear combinations of elements of a basis given by eigensections of $\theta$. As such a linear combination determines the section uniquely, we get the injectivity of $\iota$ over $U^{0}$.

The statement that $S_{\mathcal{E}_{\alpha}}^{i}$ form an $\mathbf{R}$-parabolic sheaf follows easily from the projection formula

$$
\mathcal{O}_{\left(p \circ \sigma_{i}\right)^{-1}(U)}\left(\left(p \circ \sigma_{i}\right)^{-1}\left(p_{i}\right)\right)=\left(p \circ \sigma_{i}\right)^{\star}\left(\mathcal{O}_{U}\left(p_{i}\right)\right) .
$$

We are now going to extend the above construction recursively to the case of arbitrarily high order poles $p_{i}$. Denote by $-\zeta_{i, j}^{m} / m$ the $j$ 'th eigenvalue of the matrix $A_{i}^{m}$ appearing in (1). Again by Rouché's formula, the eigenvalues $\zeta_{i, j}$ of $\theta$ are of the form

$$
\left(\frac{\zeta_{i, j}^{m_{i}}}{z_{i}^{m_{i}+1}}+\cdots+\frac{\zeta_{i, j}^{1}}{z_{i}^{2}}+O\left(z_{i}^{-2+\frac{1}{r}}\right)\right) \mathrm{d} z_{i}
$$

near $p_{i}$. Correspondingly, there exists a local splitting (17) of $\mathcal{E}$ where each direct summand consists of the direct sum of all eigenspaces of $\theta$ having the same expansion up to order $z_{i}^{-2}$. Just as in Claim 4.1, this decomposition is actually defined over $\mathbf{C}\left(\left\{z_{i}\right\}\right)$. We now construct a birational map

$$
\tilde{\sigma}: \widetilde{Z} \rightarrow Z
$$

recursively. If $m_{i}>1$ then apply a blow-up

$$
\sigma_{i, j}: Z^{i, j} \longrightarrow Z
$$


at each point $\left(p_{i}, \zeta_{i, j}^{m_{i}}\right) \in Z$ with respect to the local trivialisation

$$
\lambda_{i}=z_{i}^{-m_{i}-1} \mathrm{~d} z_{i}
$$

of $L$ near $p_{i}$. For each eigenvalue $\zeta_{i, j}^{m_{i}}$ the point $\left(p_{i}, \zeta_{i, j}^{m_{i}}\right) \in Z$ is only taken once, independently of its multiplicity as an eigenvalue. In concrete terms, using the holomorphic coordinate (14) introduce new homogeneous coordinates

$$
\left(z_{i, j}^{\prime}: \zeta_{i, j}^{\prime}\right) \in E_{i, j}=\mathbf{P}^{1}
$$

and let $U_{i, j}$ denote a neighborhood of $\left(p_{i}, \zeta_{i, j}^{m_{i}}\right)$ containing no other point $\left(p_{i^{\prime}}, \zeta_{i^{\prime}, j^{\prime}}^{m_{i^{\prime}}}\right)$ for $i^{\prime} \neq i, j^{\prime} \neq j$. Then the preimage by $\sigma_{i, j}$ of $U_{i, j}$ is given by

$$
\sigma_{i, j}^{-1}\left(U_{i, j}\right)=\left\{z_{i} \zeta_{i, j}^{\prime}=z_{i, j}^{\prime}\left(\zeta_{i}-\zeta_{i, j}^{m_{i}}\right)\right\} \subset U_{i, j} \times \mathbf{P}^{1} .
$$

Let us define an affine open chart of $Z^{i, j}$ by

$$
U_{i, j}^{\prime}=\left(z_{i, j}^{\prime} \neq 0\right) \text {. }
$$

Now just as in (23) if we denote by $\theta^{i, j}$ the restriction of $\theta$ to the $-\zeta_{i, j}^{m_{i}} / m_{i^{-}}$ eigenspace $\mathcal{E}^{i, j}$ of $A_{i}^{m_{i}}$, then the equation of the total transform of $\Sigma$ with respect to $\sigma_{i, j}$ in $U_{i, j}^{\prime}$ writes

$$
\left(\frac{1}{z_{i, j}^{\prime}}\right)^{d_{i, j}} \operatorname{det}\left(z_{i} \zeta_{i, j}^{\prime} \lambda_{i} \mathrm{I}_{\mathcal{E}^{i, j}}-z_{i, j}^{\prime} \tilde{\theta}^{i, j}\right)
$$

where $d_{i, j}$ stands for the multiplicity of $\zeta_{i, j}^{m_{i}}$ as an eigenvalue of $-m_{i} A_{i}^{m_{i}}$ and

$$
\begin{aligned}
& \tilde{\theta}^{i, j} \in\left(p \circ \sigma_{i, j}\right)^{*}\left(L\left(-p_{i}\right) \otimes \mathcal{E} n d\left(\mathcal{E}^{i, j}\right)\right) \\
& \tilde{\theta}^{i, j}=\left(\mathrm{d} \tilde{Q}_{i, j}+\Lambda_{i, j}\left(z_{i}\right)^{-1}+\text { holomorphic terms }\right) \mathrm{d} z_{i}
\end{aligned}
$$

for some

$$
\tilde{Q}_{i, j}=\tilde{A}_{i, j}^{m_{i}-1} z_{i}^{-m_{i}-1}+\cdots+\tilde{A}_{i, j}^{1} z_{i}^{-1} .
$$

Specifically, $\tilde{A}_{i, j}^{m}$ is the restriction of $A_{i, j}^{m}$ to the $\zeta_{i, j}^{m_{i}}$-eigenspace of $-m_{i} A_{i, j}^{m_{i}}$. Again, $E_{i, j}$ is a component of multiplicity $d_{i, j}$ of the total transform of $\Sigma$ by the transformation $\sigma_{i, j}$, and the proper transform is given by

$$
\operatorname{det}\left(\zeta_{i, j}^{\prime} \lambda_{i} \mathrm{I}_{\mathcal{E}^{i, j}}-z_{i, j}^{\prime} z_{i}^{-1} \tilde{\theta}^{i, j}\right) \in H^{0}\left(U_{i, j}^{\prime},\left(p \circ \sigma_{i, j}\right)^{*}\left(L \otimes \mathcal{E} n d\left(\mathcal{E}^{i, j}\right)\right) \otimes \mathcal{O}_{Z^{i, j}}\left(-E_{i, j}\right)\right) \text {. }
$$

The leading-order term of the matrix in the argument of this determinant as $z_{i} \rightarrow 0$ is

$$
\left(\zeta_{i, j}^{\prime} \mathrm{I}_{\mathcal{E}^{i, j}}+z_{i, j}^{\prime}\left(m_{i}-1\right) \tilde{A}_{i, j}^{m_{i}-1}\right) \lambda_{i}
$$

in particular, if for any $j^{\prime}$ such that $\zeta_{i, j^{\prime}}^{m_{i}}=\zeta_{i, j}^{m_{i}}$ we let

$$
\left(z_{i, j}^{\prime}: \zeta_{i, j}^{\prime}\right)=\left(1: \zeta_{i, j^{\prime}}^{m_{i}-1}\right)
$$


then the above matrix is singular. Let us introduce a further subscript to $\zeta_{i, j^{\prime}}^{m_{i}-1}$ in order to remember the information that $\zeta_{i, j^{\prime}}^{m_{i}}=\zeta_{i, j}^{m_{i}}$ : set

$$
\zeta_{i, j, j^{\prime}}^{m_{i}-1}=\zeta_{i, j^{\prime}}^{m_{i}-1}
$$

Said differently, the proper transform of $\Sigma$ by $\sigma_{i, j}$ intersects $E_{i, j}$ precisely in the points

$$
\left(1: \zeta_{i, j, j^{\prime}}^{m_{i}-1}\right)
$$

Therefore, we may recursively define a sequence of blow-ups of $Z$ by the following

\section{Construction 1. (1) we define}

$$
\sigma_{1}: Z^{1} \longrightarrow Z
$$

as the fibre product of the monodial transformations $\sigma_{i, j}$ for all $1 \leq$ $i \leq n$ with $m_{i} \geq 1$ and eigenvalues $\zeta_{i, j}^{m_{i}}$ of $-m_{i} A_{i, j}^{m_{i}}$;

(2) next we blow up $Z^{1}$ at the points on $E_{i, j}$ corresponding to the points (35) of intersection with the proper transform of $\Sigma$ along $\sigma_{1}$ (these are therefore indexed by joint eigenvalues of $A_{i}^{m_{i}}, A_{i}^{m_{i}-1}$ ) for all $i$ such that $m_{i} \geq 2$, denote the blow-up by

$$
\sigma_{2}: Z^{2} \longrightarrow Z^{1}
$$

(3) in the $(m+1)$ 'th step for all $i$ with $m_{i} \geq m$ and all eigenvalue

$$
\zeta_{i, j, j^{\prime}, \ldots, j(m)}^{m_{i}-m}=\zeta_{i, j^{(m)}}^{m_{i}-m}
$$

of $\left(m-m_{i}\right) A_{i}^{m_{i}-m}$ we blow up $Z^{m}$ at the intersection point

$$
\left(z_{i, j, j^{\prime} \ldots, j(m-1)}^{\prime}: \zeta_{i, j, j^{\prime} \ldots, j(m-1)}^{\prime}\right)=\left(1: \zeta_{i, j, j^{\prime}, \ldots, j(m)}^{m_{i}-m}\right) \in E_{i, j, j^{\prime} \ldots, j^{(m-1)}}
$$

of the proper transform of $\Sigma$ along $\sigma_{m-1}$ with the exceptional divisors of $\sigma_{m-1}$ projecting to the point $p_{i}$, call the resulting exceptional divisor

$$
E_{i, j, j^{\prime} \ldots, j^{(m)}}
$$

with homogeneous coordinates

$$
\left(z_{i, j, j^{\prime} \ldots, j(m)}^{\prime}: \zeta_{i, j, j^{\prime} \ldots, j(m)}^{\prime}\right)
$$

and set

$$
U_{i, j, j^{\prime} \ldots, j^{(m)}}^{\prime}=\left(z_{i, j, j^{\prime} \ldots, j^{(m)}}^{\prime} \neq 0\right) \subset Z^{m} ;
$$

(4) finally we define (29) as $Z^{M}$ for the value $M=\max _{1 \leq i \leq n}\left(m_{i}\right)$ with

$$
\tilde{\sigma}=\sigma_{1} \circ \cdots \circ \sigma_{M} \text {. }
$$


Notice that the degree of $\tilde{Q}_{i, j}$ appearing in (34) is one less than that of $Q_{i}$; similarly, in the $(m+1)$ 'th step of the recursive procedure the proper transform of $\Sigma$ in a local chart is defined by a section

$$
\operatorname{det}\left(\zeta_{i, j, j^{\prime}, \ldots, j(m)}^{\prime} \lambda_{i} \mathrm{I}_{\mathcal{E}^{i, j, j^{\prime}, \ldots, j}(m)}-z_{i, j, j^{\prime}, \ldots, j(m)}^{\prime} z_{i}^{-m} \tilde{\theta}^{i, j, j^{\prime}, \ldots, j^{(m)}}\right)
$$

for some

$$
\begin{aligned}
& \tilde{\theta}^{i, j, j^{\prime}, \ldots, j^{(m)}}=\mathrm{d} \tilde{Q}_{i, j, j^{\prime}, \ldots, j(m)}+\left(\Lambda_{i, j, j^{\prime}, \ldots, j(m)}\left(z_{i}\right)^{-1}+\text { holomorphic terms }\right) \mathrm{d} z_{i} \\
& \tilde{Q}_{i, j, j^{\prime}, \ldots, j(m)}=A_{i, j, j^{\prime}, \ldots, j(m)}^{m_{i}-m-1} z_{i}^{-m_{i}+m+1}+\cdots+A_{i, j, j^{\prime}, \ldots, j(m)}^{1} z_{i}^{-1}
\end{aligned}
$$

where the matrices on the right hand side of the latter equation are restrictions of $A_{i}^{m_{i}-m-1}, \ldots, A_{i}^{1}, \Lambda_{i}$ to joint eigenspaces of

$$
-m_{i} A_{i}^{m_{i}}, \ldots,\left(m-m_{i}\right) A_{i}^{m_{i}-m}
$$

for the eigenvalues

$$
\zeta_{i, j}^{m_{i}}, \zeta_{i, j, j^{\prime}}^{m_{i}-1}, \ldots, \zeta_{i, j, j^{\prime}, \ldots, j(m)}^{m_{i}-m}
$$

respectively. We will denote the dimension of this joint eigenspace by

$$
d_{i, j, j^{\prime}, \ldots, j(m)} .
$$

Now just as in (25), the map $\tilde{\theta}^{i, j, j^{\prime}, \ldots, j^{\left(m_{i}-1\right)}}$ is of the form

$$
z_{i}^{-m_{i}} \tilde{\theta}^{i, j, j^{\prime}, \ldots, j^{\left(m_{i}-1\right)}}=\left(\Lambda_{i, j, j^{\prime}, \ldots, j}\left(m_{\left.i^{-1}\right)}+O\left(z_{i}\right)\right) \lambda_{i},\right.
$$

hence it gives rise to maps

$$
z_{i}^{-m_{i}} \tilde{\theta}_{\alpha}^{i, j, j^{\prime}, \ldots, j^{\left(m_{i}-1\right)}}:(p \circ \tilde{\sigma})^{\star} \mathcal{E}_{\alpha}^{i, j, j^{\prime}, \ldots, j^{\left(m_{i}-1\right)}} \otimes L^{\vee} \longrightarrow(p \circ \tilde{\sigma})^{\star} \mathcal{E}_{\alpha}^{i, j, j^{\prime}, \ldots, j^{\left(m_{i}-1\right)}}
$$

for every $\alpha \in \mathbf{R}$ and $i, j, j^{\prime}, \ldots, j^{\left(m_{i}-1\right)}$, where $\mathcal{E}_{\alpha}^{i, j, j^{\prime}, \ldots, j^{(m)}}$ stands for the filtration on the corresponding simultaneous eigenspace obtained from the parabolic structure by virtue of Claim 2.1. Now just as we refined the formula (24) defining the proper transform of the spectral curve to define a parabolic sheaf (26) we may again refine (37) to define coherent sheaves on

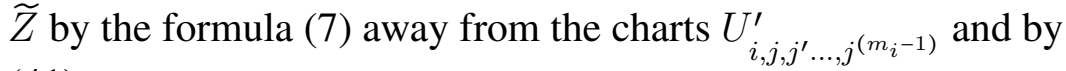

$$
S_{\mathcal{E}_{\alpha}}=\operatorname{coker}\left(\zeta_{i, j, j^{\prime}, \ldots, j^{\left(m_{i}-1\right)}}^{\prime} \lambda_{i} \mathrm{I}_{\mathcal{E}_{\alpha}^{i, j, j^{\prime}, \ldots, j}\left(m_{i}-1\right)}-z_{i, j, j^{\prime}, \ldots, j^{\left(m_{i}-1\right)}}^{\prime} z_{i}^{-m_{i}} \tilde{\theta}_{\alpha}^{i, j, j^{\prime}, \ldots, j^{\left(m_{i}-1\right)}}\right)
$$

on $U_{i, j, j^{\prime} \ldots, j}^{\prime}{\left(m_{i}-1\right)}$, the argument being a section of the bundle

$$
\begin{aligned}
(p \circ \tilde{\sigma})^{*} & \left(\mathcal{E} n d\left(\mathcal{E}_{\alpha}^{i, j, j^{\prime}, \ldots, j^{\left(m_{i}-1\right)}}\right) \otimes L\right) \otimes \tilde{\sigma}^{*} \mathcal{O}_{Z}(1) \\
& \otimes \mathcal{O}_{\widetilde{Z}}\left(-E_{i, j, j^{\prime}, \ldots, j^{\left(m_{i}-1\right)}}\right) .
\end{aligned}
$$

Now, consider the groupoid

$$
\mathcal{H} \text { iggs }\left(C, r,\left\{p_{i}\right\},\left\{m_{i}\right\},\left\{A_{i}^{m}\right\}\right)
$$


whose objects are parabolic Higgs bundles of rank $r$ on $C$ with for all $1 \leq i \leq$ $n$ an irregular singularity with pole of order $m_{i}$ at $p_{i}$ locally of the form (3) with $Q_{i}$ of the form (1) for some semi-simple matrices $A_{i}^{m}$, endowed with a compatible parabolic structure, and whose morphisms $\operatorname{Hom}_{\mathcal{H} i g g s}\left((\mathcal{E}, \theta),\left(\mathcal{E}^{\prime}, \theta^{\prime}\right)\right)$ are isomorphisms of such parabolic Higgs bundles, i.e. isomorphisms of parabolic vector bundles

$$
\phi_{\alpha}: \mathcal{E}_{\alpha} \stackrel{\sim}{\rightarrow} \mathcal{E}_{\alpha}^{\prime}
$$

for every $\alpha \in \mathbf{R}$ such that

$$
\left.\phi_{\alpha}\right|_{\mathcal{E}_{\alpha^{\prime}}}=\phi_{\alpha^{\prime}}
$$

for all $\alpha^{\prime}>\alpha$ and

$$
\left(\phi_{\alpha} \otimes \operatorname{Id}_{L}\right) \circ \theta=\theta^{\prime} \circ \phi_{\alpha} .
$$

On the other hand, consider the groupoid

$$
\operatorname{Sheaf}\left(\widetilde{Z}, r,(p \circ \tilde{\sigma})^{-1}\left(D_{\text {red }}\right)\right)
$$

whose objects are $\mathbf{R}$-parabolic pure sheaves $S_{\alpha}$ of dimension 1 and rank 1 with parabolic divisor

$$
(p \circ \tilde{\sigma})^{-1}\left(D_{\text {red }}\right)=(p \circ \tilde{\sigma})^{-1}\left(\sum_{i} p_{i}\right)
$$

on $\widetilde{Z}$, with support $\widetilde{\Sigma}$ satisfying the following properties:

(1) $\widetilde{\Sigma}$ is generically $r$ to 1 over $C$,

(2) $\widetilde{\Sigma} \cap(\xi=0)=\varnothing$,

(3) for all $1 \leq i \leq n$ we have $\widetilde{\Sigma} \cap E_{i, j, j^{\prime}, \ldots, j^{\left(m_{i}-1\right)}} \subset U_{i, j, j^{\prime}, \ldots, j^{\left(m_{i}-1\right)}}^{\prime}$, i.e. the support does not pass through the point at infinity of the exceptional divisor $E_{i, j, j^{\prime}, \ldots, j\left(m_{i}-1\right)}$,

(4) for all $1 \leq i \leq n$ the intersection $\widetilde{\Sigma} \cap E_{i, j, j^{\prime}, \ldots, j^{\left(m_{i}-1\right)}}$ consists of $d_{i, j, j^{\prime}, \ldots, j^{\left(m_{i}-1\right)}}$ points counted with multiplicity,

(5) for all $1 \leq i \leq n$ and all $m<m_{i}$ the proper transform of $E_{i, j, j^{\prime}, \ldots, j}(m-1)$ in $\widetilde{Z}$ does not intersect $\widetilde{\Sigma}$.

The morphisms $\operatorname{Hom}_{\mathcal{S} h e a f}\left(S_{\bullet}, S_{\bullet}^{\prime}\right)$ are defined to be the isomorphisms of parabolic sheaves, i.e. for each $\alpha \in \mathbf{R}$ an isomorphism

$$
\psi_{\alpha}: S_{\alpha} \stackrel{\sim}{\rightarrow} S_{\alpha}^{\prime}
$$

such that for all $\alpha^{\prime}>\alpha$ we have

$$
\left.\psi_{\alpha}\right|_{S_{\alpha^{\prime}}}=\psi_{\alpha^{\prime}} .
$$

Notice that this category depends on the choice of semi-simple linear endomorphisms $A_{i}^{m}$ through the surface $\widetilde{Z}$.

The results of this section can be summarized as follows. 
Theorem 4.3. The functor

$$
\begin{aligned}
\mathcal{H} i g g s\left(C, r,\left\{p_{i}\right\},\left\{m_{i}\right\},\left\{A_{i}^{m}\right\}\right) & \rightarrow \operatorname{Sheaf}\left(\widetilde{Z}, r,(p \circ \tilde{\sigma})^{-1}\left(D_{\text {red }}\right)\right) \\
\left(\mathcal{E}_{\bullet}, \theta\right) & \mapsto S_{\mathcal{E}_{\bullet}}
\end{aligned}
$$

given by (41) establishes an isomorphism of categories.

Proof. The sheaves $S_{\mathcal{E}}$ satisfy property (1) because $\mathcal{E}$ is of rank $r$. Furthermore as $\widetilde{\Sigma}$ is the spectral curve of a regular map

$$
\mathcal{E} \rightarrow \mathcal{E} \otimes K_{C}(D)
$$

it stays in the affine subset $\xi \neq 0$ of $Z$. By (40) $\widetilde{\Sigma}$ intersects the exceptional divisor $E_{i, j, j^{\prime}, \ldots, j\left(m_{i}-1\right)}$ in the points

$$
\left(z_{i, j, j^{\prime} \ldots, j\left(m_{i}-1\right)}^{\prime}: \zeta_{i, j, j^{\prime} \ldots, j^{\left(m_{i}-1\right)}}^{\prime}\right)=\left(1: \lambda_{i, j, j^{\prime}, \ldots, j^{\left(m_{i}-1\right)}, j^{m_{i}}}\right)
$$

where the $\lambda_{\ldots}$ on the right hand side stand for the eigenvalues of $\Lambda_{i}$ restricted to the joint eigenspaces of $A_{i}^{m_{i}}, \ldots, A_{i}^{1}$, and this shows (3) and (4). Next, (5) holds because to define $\widetilde{Z}$ in Construction 1 we blew up all points (36) of intersection of the proper transform of $\Sigma$ with $E_{i, j, j^{\prime}, \ldots, j(m-1)}$ and the support of the sheaves $S_{\mathcal{E}_{\bullet}}$ is the proper transform $\widetilde{\Sigma}$. Purity of $S_{\mathcal{E}_{\bullet}}$ follows the exact same argument as in the case $m_{i}=1$ (c.f. Claim 4.2) based on the Auslander-Buchsbaum formula. Finally, again as in the case $m_{i}=1$ the identity

$$
(p \circ \tilde{\sigma})^{*} \mathcal{O}_{C}\left(D_{\text {red }}\right)=\mathcal{O}_{\widetilde{Z}}\left((p \circ \tilde{\sigma})^{-1}\left(D_{\text {red }}\right)\right)
$$

immediately shows that the sheaves $S_{\mathcal{E}_{\bullet}}$ are $\mathbf{R}$-parabolic with divisor $(p \circ$ $\tilde{\sigma})^{-1}\left(D_{\text {red }}\right)$.

The inverse is the direct image functor

$$
\mathcal{E}_{\alpha}=(p \circ \sigma)_{\star} S_{\mathcal{E}_{\alpha}}, \quad \theta_{\alpha}=\pi_{*}(\zeta \cdot)
$$

by a straightforward generalisation of Claim 4.2 to the case of order $m_{i} \geq 2$. There remains to check that

- the irregular part of $\theta$ is of the form (3), (1) and

- that the residue $\Lambda_{i}$ of $\theta$ at $z_{i}=0$ respects the parabolic filtration of $\mathcal{E}_{\text {. at }} p_{i}$.

For the proof of these statements observe that by the construction of $\mathcal{E}_{\alpha}$ it has a local splitting

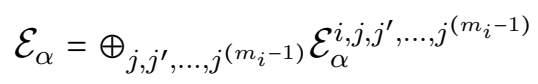

according to the support of sections of $S_{\mathcal{E}_{\alpha}}$ : local sections of $\mathcal{E}_{\alpha}^{i, j, j^{\prime}, \ldots, j^{\left(m_{i}-1\right)}}$ are defined as the push-forward of local sections of $S_{\mathcal{E}_{\alpha}}$ supported on the 
branches of $\widetilde{\Sigma}$ intersecting a given exceptional divisor $E_{i, j, j^{\prime}, \ldots, j^{\left(m_{i}-1\right)}}$. From (32), on $U_{i, j}^{\prime}$ we deduce the identity

$$
\zeta_{i}=\zeta_{i, j}^{m_{i}}+z_{i} \frac{\zeta_{i, j}^{\prime}}{z_{i, j}^{\prime}}
$$

Here by the definition of $U_{i, j}^{\prime}$ we may normalize $z_{i, j}^{\prime}=1$. In the case $m_{i}>1$ Construction 1 proceeds by applying a blow-up $\sigma_{i, j, j^{\prime}}$ at the point $\zeta_{i, j}^{\prime}=$ $\zeta_{i, j, j^{\prime}}^{m_{i}-1}$ of $E_{i, j}$ given by the formula

$$
z_{i} \zeta_{i, j, j^{\prime}}^{\prime}=z_{i, j, j^{\prime}}^{\prime}\left(\zeta_{i, j}^{\prime}-\zeta_{i, j, j^{\prime}}^{m_{i}-1}\right)
$$

We may again set $z_{i, j, j^{\prime}}^{\prime}=1$ on $U_{i, j, j^{\prime}}^{\prime}$ and solve this expression for $\zeta_{i, j}^{\prime}$ :

$$
\zeta_{i, j}^{\prime}=\zeta_{i, j, j^{\prime}}^{m_{i}-1}+z_{i} \zeta_{i, j, j^{\prime}}^{\prime}
$$

Plugging this into (44) yields

$$
\zeta_{i}=\zeta_{i, j}^{m_{i}}+z_{i} \zeta_{i, j, j^{\prime}}^{m_{i}-1}+z_{i}^{2} \zeta_{i, j, j^{\prime}}^{\prime}
$$

By induction on $m_{i}$, this argument shows on $U_{\left.i, j, j^{\prime} \ldots, j^{(} m_{i}-1\right)}^{\prime}$ (using the normalization $\left.z_{i, j, j^{\prime} \ldots, j}^{\prime}\left(m_{i}-1\right)=1\right)$ the identity

$$
\zeta_{i}=\zeta_{i, j}^{m_{i}}+z_{i} \zeta_{i, j, j^{\prime}}^{m_{i}-1}+\cdots+z_{i}^{m_{i}-1} \zeta_{i, j, j^{\prime} \ldots, j^{\left(m_{i}-1\right)}}^{1}+z_{i}^{m_{i}} \zeta_{i, j, j^{\prime} \ldots, j^{\left(m_{i}-1\right)}}^{\prime}
$$

Here $\zeta_{i, j}^{m_{i}}, \ldots, \zeta_{i, j, j^{\prime} \ldots, j\left(m_{i}-1\right)}^{1}$ are constants, therefore the restriction to $\mathcal{E}_{\alpha}^{i, j, j^{\prime}, \ldots, j^{\left(m_{i}-1\right)}}$ of the direct image of the multiplication map by $\zeta=\zeta_{i} \lambda_{i}$ has the expansion

$$
\left(\frac{\zeta_{i, j}^{m_{i}}}{z_{i}^{m_{i}+1}}+\cdots+\frac{\zeta_{i, j}^{1}}{z_{i}^{2}}\right) \operatorname{Id}_{\mathcal{E}_{\alpha}^{i, j, j^{\prime}, \ldots, j}\left(m_{i}-1\right)} \mathrm{d} z+O\left(z_{i}^{-2+\frac{1}{r}}\right) \mathrm{d} z
$$

which is precisely the first assertion. Furthermore, the residue of $\theta$ at $p_{i}$ is obtained as the direct image of the multiplication map by

$$
\zeta_{i, j, j^{\prime} \ldots, j}^{\prime}\left(m_{i}-1\right)
$$

which is a local coordinate of the surface $\widetilde{Z}$. As $S_{\mathcal{E}_{\alpha}}$ is a sheaf of $\mathcal{O}_{\widetilde{Z}^{-}}$ modules, it is clearly preserved by multiplication by $\zeta_{i, j, j^{\prime} \ldots, j}^{\prime}\left(m_{i}-1\right)$. Therefore $\operatorname{res}_{p_{i}}(\theta)$ preserves $\mathcal{E}_{\alpha}$, which is the second assertion.

\section{POISSON ISOMORPHISM}

In this section we prove that the natural holomorphic Poisson structures on the moduli spaces associated to the groupoids appearing in Theorem 4.3 are preserved by the correspondence of the Theorem. The proof closely follows that of Proposition 5.1 [24]. We start by defining these Poisson structures. 
5.1. Irregular Dolbeault moduli space. Let us first treat the moduli space of stable irregular parabolic Higgs bundles on $C$ with fixed semi-simple irregular part (3), (1) and residue in a fixed regular orbit: by [5] it carries an Atiyah-Bott hyper-Kähler structure, in particular for the Dolbeault holomorphic structure $I$ it admits a holomorphic symplectic structure. These irregular Dolbeault moduli spaces $M_{\text {Dol }}^{\text {irr }}$ may then be put into a family

$$
\mathcal{M}_{\text {Dol }}^{\mathrm{irr}} \rightarrow \mathbf{C}^{r \sum_{i}\left(m_{i}+1\right)}
$$

by varying the eigenvalues of the matrices $A_{i}^{m}$ and of $\Lambda_{i}$ arbitrarily. In concrete terms, $\mathcal{M}_{\text {Dol }}^{\text {irr }}$ represents the functor from Artinian schemes over $\mathbf{C}$ to sets mapping

- a scheme $T$ to the set of isomorphism classes of parabolically stable pairs $(\overline{\mathcal{E}}, \bar{\theta})$ where $\overline{\mathcal{E}}$ is a regular vector bundle over $T \times C$ and

$$
\bar{\theta} \in H^{0}\left(T \times C, \mathcal{E} n d(\overline{\mathcal{E}}) \otimes_{\mathcal{O}_{T \times C}} p_{C}^{*} L\right),
$$

with $p_{C}: T \times C \rightarrow C$ the projection map;

- and a morphism $S \rightarrow T$ of such schemes to the set of maps of Higgs bundles parameterized by $S$ and $T$ respectively.

The condition of parabolic stability is a parabolic version of slope-stability: one first introduces the notion of parabolic degree as the sum of the usual degree and all the parabolic weights, and then induces parabolic weights and parabolic degree on sub-objects; for further details see [7]. The map in (45) associates to $(\mathcal{E}, \theta)$ the eigenvalues of the polar part of $\theta$ considered as a meromorphic section of $\mathcal{E} n d(\mathcal{E}) \otimes_{\mathcal{O}_{C}} K_{C}$.

Remark 5.1. (1) To the knowledge of the author, this irregular Dolbeault moduli functor has not yet been studied algebraically. Notice nonetheless that E. Markman studied in [18] a related moduli problem, which is essentially the same as our functor above except that there the parabolic structure is not present and the irregular type may be twisted (non-semi-simple).

(2) An analogous de Rham moduli space is constructed algebraically in [2]; however, as it is explained in Remark $1.2 \mathrm{op}$. cit., the data they fix at the singularities is somewhat different from the usual irregular type, and this latter cannot be determined from it. On the other hand, Inaba and Saito consider more general families of puctured curves

$$
\mathcal{C} \rightarrow T
$$

instead of just a product.

(3) Depending on the eigenvalues of the singular parts, the moduli space $M_{\mathrm{Dol}}^{\mathrm{irr}}$ might be empty. For instance, as it readily follows from the residue theorem, this will be the case unless the sum of 
the traces of all the residues is an integer $-\delta$. In this case, we have $\operatorname{deg}(\mathcal{E})=\delta$. However, the above consequence of the residue theorem is just a first easy condition in deciding whether or not the moduli space is empty - in general, this problem, called irregular Deligne-Simpson problem is hard. The correspondence of Theorem 4.3 holds (but is vacuous) even if the irregular moduli space is empty.

Elements of $\mathbf{C}^{r \sum_{i}\left(m_{i}+1\right)}$ will be denoted as nested sequeces of eigenvalues

$$
\left(\zeta_{i, j}^{m_{i}}, \ldots, \zeta_{i, j, j^{\prime} \ldots, j^{\left(m_{i}-1\right)}}^{1}, \lambda_{i, j, j^{\prime} \ldots, j^{\left(m_{i}\right)}}\right)_{i, j, j^{\prime} \ldots, j^{\left(m_{i}\right)}}
$$

where the $\zeta_{i, j, j^{\prime} \ldots, j^{(m)}}^{m}$ denote eigenvalues of $-m A_{i}^{m}$ for $0 \leq m<m_{i}$ and $\lambda_{i, j, j^{\prime} \ldots, j\left(m_{i}\right)}$ is an eigenvalue of $\Lambda_{i, j, j^{\prime}, \ldots, j^{\left(m_{i}-1\right)}}$ (42). One could equally let the parabolic weights $\alpha_{i}^{l}$ vary and this would give further deformation parameters, but we will ignore this point here. The smooth part of $\mathcal{M}_{\text {Dol }}^{\mathrm{irr}}$ is a holomorphic Poisson manifold with Poisson bivector field denoted by $\Pi_{\mathrm{Dol}}$. Let us be more specific concerning this holomorphic Poisson structure, following [6] where the non-singular case is treated. It is easy to see that the deformation theory of an irregular Higgs bundle $(\mathcal{E}, \theta)$ in $\mathcal{M}_{\mathrm{Dol}}^{\mathrm{irr}}$ is governed by the hypercohomology spaces $\mathbf{H}^{\bullet}$ of the complex

$$
\mathcal{E} n d(\mathcal{E}) \stackrel{\operatorname{ad}_{\theta}}{\longrightarrow} \mathcal{E} n d(\mathcal{E}) \otimes L
$$

with $L=K_{C}(D)$. Infinitesimal deformations are given by the first hypercohomology space $\mathbf{H}^{1}$ and $\mathbf{H}^{2}$ is the obstruction space. By Grothendieck duality, the cotangent space of (45) is then given by the first hypercohomology of the complex

$$
\mathcal{E} n d(\mathcal{E}) \otimes \mathcal{O}_{C}(-D) \stackrel{\mathrm{ad}_{\theta}}{\longrightarrow} \mathcal{E} n d(\mathcal{E}) \otimes K_{C}
$$

Given two cotangent vectors

$$
[T],[X] \in \mathbf{H}^{1}\left(\mathcal{E} n d(\mathcal{E}) \otimes \mathcal{O}_{C}(-D) \rightarrow \mathcal{E} n d(\mathcal{E}) \otimes K_{C}\right)
$$

represented by endomorphism-valued 1-forms $T, X$ their cup product

$$
[T] \cup[X]=[T \wedge X]
$$

belongs to the second hypercohomology group of the complex

$$
C^{0} \stackrel{\left(\operatorname{ad}_{\theta} \otimes \operatorname{Id}, \mathrm{Id} \otimes \mathrm{ad}_{\theta}\right)}{\longrightarrow} C^{1} \stackrel{\mathrm{Id} \otimes \mathrm{ad}_{\theta}-\mathrm{ad}_{\theta} \otimes \mathrm{Id}}{\longrightarrow} C^{2}
$$


with

$$
\begin{aligned}
C^{0}= & \left(\mathcal{E} n d(\mathcal{E}) \otimes \mathcal{O}_{C}(-D)\right) \otimes\left(\mathcal{E} n d(\mathcal{E}) \otimes \mathcal{O}_{C}(-D)\right) \\
C^{1}= & {\left[\left(\mathcal{E} n d(\mathcal{E}) \otimes K_{C}\right) \otimes\left(\mathcal{E} n d(\mathcal{E}) \otimes \mathcal{O}_{C}(-D)\right)\right] } \\
& \oplus\left[\left(\mathcal{E} n d(\mathcal{E}) \otimes \mathcal{O}_{C}(-D)\right) \otimes\left(\mathcal{E} n d(\mathcal{E}) \otimes K_{C}\right)\right] \\
C^{2}= & \left(\mathcal{E} n d(\mathcal{E}) \otimes K_{C}\right) \otimes\left(\mathcal{E} n d(\mathcal{E}) \otimes K_{C}\right) .
\end{aligned}
$$

The ad-invariant symmetric bilinear form on $\mathrm{Gl}_{r}$

$$
B: \varphi, \psi \mapsto \operatorname{tr}(\varphi \psi)
$$

induces a chain map $\Phi$ from (47) to

$$
0 \rightarrow K_{C}(-D) \rightarrow 0 .
$$

In concrete terms, to a cocycle

$$
\left(\varphi_{1} \otimes \psi_{1}, \psi_{2} \otimes \varphi_{2}\right) \in C^{1}
$$

the map $\Phi$ associates the section

$$
B\left(\varphi_{1}, \psi_{1}\right)+B\left(\psi_{2}, \varphi_{2}\right) .
$$

Plainly $\Phi$ is a chain map because the bilinear form $B$ is ad-invariant

$$
B\left(\operatorname{ad}_{\theta}(\eta), \zeta\right)+B\left(\eta, \operatorname{ad}_{\theta}(\zeta)\right)=0 .
$$

It then follows that the image of $[T] \cup[X]$ by $\Phi$ defines a degree 2 hypercohomology class in (49), i.e. a class in

$$
H^{1}\left(C, K_{C}(-D)\right) \cong\left(H^{0}(C, \mathcal{O}(D))\right)^{\vee} .
$$

The dual of the vector space on the right hand side fits into a short exact sequence

$$
0 \rightarrow H^{0}(C, \mathcal{O}) \rightarrow H^{0}(C, \mathcal{O}(D)) \rightarrow \oplus_{i=1}^{n} \mathbf{C}_{p_{i}}^{m_{i}+1} \rightarrow 0,
$$

where $\mathbf{C}_{p}$ stands for the skyscraper sheaf supported at the point $p \in C$. In particular, $H^{0}(C, \mathcal{O}(D))$ contains the element $1 \in H^{0}(C, \mathcal{O})$, hence any element in (50) can be evaluated on this class. We may therefore define an alternating bilinear map by

$$
\begin{aligned}
\Pi_{\text {Dol }}: T^{*} \mathcal{M}_{\text {Dol }}^{\mathrm{irr}} \times T^{*} \mathcal{M}_{\text {Dol }}^{\mathrm{irr}} & \rightarrow \mathbf{C} \\
([T],[X]) & \mapsto\langle\Phi([T] \cup[X]), 1\rangle
\end{aligned}
$$

where $\langle.,$.$\rangle stands for Serre duality (50). As usual, this formula is the re-$ duction of an infinite-dimensional flat pairing on an $L^{2}$-space of 1-forms with values in the endomorphisms of the smooth vector bundle underlying $\mathcal{E}$, so the Schouten-bracket $\left[\Pi_{\text {Dol }}, \Pi_{\text {Dol }}\right]$ of $\Pi_{\text {Dol }}$ with itself is 0 . Since using the Dolbeault resolution of $K_{C}$ Serre duality is defined by integration 
of 2 -forms on $C$ and $\Phi$ is given by (48) we infer that the restriction of this pairing to the symplectic leaves reads

$$
\int_{C} \operatorname{tr}(T \wedge X)
$$

which is the usual Atiyah-Bott holomorphic symplectic form. Therefore, $\Pi_{\text {Dol }}$ defines the holomorphic Poisson structure we were looking for. Let us point out that the vector spaces in (51) localised at the points $p_{i}$ correspond to the infinitesimal modifications of some Casimir operators, i.e. to tangent vectors of the parameter space $\mathbf{C}^{r \sum_{i}\left(m_{i}+1\right)}$.

Remark 5.2. The Poisson structure $\Pi_{\text {Dol }}$ essentially matches up with that of the Main Theorem of [18] corresponding to the section

$$
1 \in H^{0}\left(C, \mathcal{O}_{C}(D)\right) \cong H^{0}\left(C, L \otimes K^{-1}\right) .
$$

5.2. Relative Picard bundles. We now turn our attention to the category of sheaves on $\widetilde{Z}$ satisfying the properties (1)-(5) of Theorem 4.3. Observe first that $Z$ is a Poisson surface for the canonical Liouville 2 -form on the total space of the canonical line bundle $K_{C}$. The degeneracy divisor of this Poisson structure on $Z$ is given by

$$
\pi^{-1} D+2(\xi)
$$

Therefore, the pull-back of this 2-form to $\widetilde{Z}$ by $\tilde{\sigma}$ also defines a Poisson structure. Let us determine its degeneracy divisor: by differentiating (32) twice (and as usual setting $z_{i, j}^{\prime}=1$ on $U_{i, j}^{\prime}$ ) one easily derives the formula

$$
z_{i} \mathrm{~d} z_{i} \wedge \mathrm{d} \zeta_{i, j}^{\prime}=\mathrm{d} z_{i} \wedge \mathrm{d} \zeta_{i}
$$

As we have already noticed after (21), on $U_{i, j}^{\prime}$ the equation $z_{i}=0$ defines precisely the exceptional divisor $E_{i, j}$. By an abuse of notation let $\left.F\right|_{p_{i}}$ and $E_{i, j, j^{\prime}, \ldots, j^{(m-1)}}$ denote the proper transforms of the fibre $\left.F\right|_{p_{i}}$ and of the exceptional divisor $E_{i, j, j^{\prime}, \ldots, j(m-1)}$ with respect to the various iterated blow-ups of Construction 1. (For the exceptional divisors this is obviously only applicable for $\sigma_{m^{\prime}}$ with $m^{\prime}>m$.) We infer that the pull-back by $\sigma_{i, j}$ of the canonical 2-form has a pole of order one less on $E_{i, j}$ than on $\left.F\right|_{p_{i}}$. Now by an easy induction argument we can show that the pull-back by $\sigma_{1} \circ \sigma_{2}$ of the canonical 2-form has a pole of order 2 less on $E_{i, j, j^{\prime}}$ than on $\left.F\right|_{p_{i}}$, and so on, the pull-back by $\sigma_{1} \circ \cdots \circ \sigma_{m_{i}}$ of the canonical 2-form has a pole of order $m_{i}$ less on $E_{i, j, j^{\prime}, \ldots, j^{\left(m_{i}-1\right)}}$ than on $\left.F\right|_{p_{i}}$. It follows that the degeneracy divisor of 
the pull-back of the canonical Poisson structure of $Z$ to $\widetilde{Z}$ is given by

$$
D_{\infty}=\pi^{-1} D+2(\xi)-\left(\sum_{i, j} E_{i, j}+\left(\sum_{j^{\prime}} 2 E_{i, j, j^{\prime}}+\left(\cdots+\sum_{j\left(m_{i}-1\right)} m_{i} E_{i, j, j^{\prime}, \ldots, j^{\left(m_{i}-1\right)}}\right) \cdots\right)\right),
$$

$$
=2(\xi)+\left.\sum_{i}\left(m_{i}+1\right) F\right|_{p_{i}}+\left(\sum_{j} m_{i} E_{i, j}+\left(\sum_{j^{\prime}}\left(m_{i}-1\right) E_{i, j, j^{\prime}}+\left(\cdots+\sum_{j\left(m_{i}-1\right)} E_{\left.\left.\left.i, j, j^{\prime}, \ldots, j^{\left(m_{i}-1\right)}\right) \cdots\right)\right)}\right.\right.\right.
$$

Consider first the Hilbert scheme

$$
\operatorname{Hilb}(\widetilde{Z}, H)
$$

of curves on $\widetilde{Z}$ with a given Hilbert polynomial $H$. Specifically, the Picard group of $\widetilde{Z}$ is generated by the fibre class $F$, the class of the infinity section $C_{\infty}$ and the classes of the exceptional divisors $E_{i, j, j^{\prime}, \ldots, j(m-1)}$ of the blowups $\sigma_{1}, \ldots, \sigma_{M}$. An ample line bundle on $\widetilde{Z}$ is given by

$$
\mathcal{L}=\mathcal{O}_{\widetilde{Z}}\left(-\sum_{m, i, j, j^{\prime}, \ldots, j(m-1)} E_{i, j, j^{\prime}, \ldots, j(m-1)}\right) \otimes \tilde{\sigma}^{*} \mathcal{O}_{Z}(1) \otimes(p \circ \tilde{\sigma})^{*} \mathcal{O}_{C}(1) .
$$

The intersection form on the second homology of $\widetilde{Z}$ is non-degenerate and we may consider the homology class dual to the class of the divisor

$$
r F+\sum_{i, j, j^{\prime}, \ldots, j^{\left(m_{i}-1\right)}} d_{i, j, j^{\prime}, \ldots, j^{\left(m_{i}-1\right)}} E_{i, j, j^{\prime}, \ldots, j^{\left(m_{i}-1\right)}}
$$

where we recall that $d_{i, j, j^{\prime}, \ldots, j^{\left(m_{i}-1\right)}}$ was defined in (39) as the dimension of the simultaneous eigenspace of the matrices $-m_{i} A_{i}^{m_{i}}, \ldots,-A_{i}^{1}$ for the eigenvalues

$$
\zeta_{i, j}^{m_{i}}, \zeta_{i, j, j^{\prime}}^{m_{i}-1}, \ldots, \zeta_{i, j}^{1}
$$

respectively. The generic curve in this class will then intersect the generic fiber of $\widetilde{Z}$ in $r$ points, the exceptional divisor $E_{i, j, j^{\prime}, \ldots, j^{\left(m_{i}-1\right)}}$ in $d_{i, j, j^{\prime}, \ldots, j}\left(m_{i^{-1}}\right)$ points counted with multiplicity, and will be disjoint from $C_{\infty}$ and the exceptional divisors $E_{i, j, j^{\prime}, \ldots, j(m-1)}$ with $m<m_{i}$. In different terms, such a curve satisfies the conditions (1)-(5) of Theorem 4.3. We then pick $H$ to be the Hilbert polynomial with respect to $\mathcal{L}$ of a curve in this class. Notice that the generic curve in this family intersects $E_{i, j, j^{\prime}, \ldots, j}\left(m_{i^{-1}}\right)$ in $d_{i, j, j^{\prime}, \ldots, j^{\left(m_{i}-1\right)}}$ distinct points, and is smooth. Let us denote by $B$ the subscheme of (53) parameterizing smooth curves. Notice however that the curves having nodal singularities at some points of $E_{i, j, j^{\prime}, \ldots, j^{\left(m_{i}-1\right)}}$ are also of interest, for they correspond via Theorem 4.3 to non-regular residues $\Lambda_{i, j, j^{\prime}, \ldots, j}\left(m_{i}-1\right)$ of the Higgs field. 
Next, let us consider the compactified Picard variety [3] corresponding to the family (53)

$$
\operatorname{Pic}_{\text {rel }}(\widetilde{Z}, H, d) \rightarrow \operatorname{Hilb}(\widetilde{Z}, H)
$$

parameterizing torsion-free coherent sheaves of rank 1 and degree $d$ on the fibers of (53). It follows from [10] that the restriction

$$
\operatorname{Pic}_{\text {rel }}^{0}(\widetilde{Z}, H, d) \rightarrow B
$$

of (54) to the space $B$ parameterizing smooth connected curves carries a canonical holomorphic Poisson structure with Poisson bivector field denoted by $\Pi_{\mathrm{Pic}^{0}}$. Let us describe explicitly $\Pi_{\mathrm{Pic}^{0}}$ : for this purpose, notice that the Zariski tangent space of $\operatorname{Pic}_{\text {rel }}^{0}(\widetilde{Z}, d, H)$ at a given sheaf $S$ is given by $\operatorname{Ext}_{\mathcal{O}_{\widetilde{Z}}}^{1}(S, S)$. By Grothendieck-duality, the Zariski cotangent space is then isomorphic to

$$
\operatorname{Ext}_{\mathcal{O}_{\widetilde{Z}}}^{1}\left(S, K_{\widetilde{Z}} \otimes S\right) \cong \operatorname{Ext}_{\mathcal{O}_{\widetilde{Z}}}^{1}\left(K_{\widetilde{Z}}^{\vee} \otimes S, S\right) .
$$

Thus the Yoneda product

$$
\operatorname{Ext}_{\mathcal{O}_{\widetilde{Z}}}^{1}\left(K_{\widetilde{Z}}^{\vee} \otimes S, S\right) \times \operatorname{Ext}_{\mathcal{O}_{\widetilde{Z}}}^{1}\left(S, K_{\widetilde{Z}} \otimes S\right) \rightarrow \operatorname{Ext}_{\mathcal{O}_{\widetilde{Z}}}^{2}\left(K_{\widetilde{Z}}^{\vee} \otimes S, K_{\widetilde{Z}} \otimes S\right)
$$

induces by duality an alternating map

$$
\operatorname{Ext}_{\mathcal{O}_{\widetilde{Z}}}^{2}\left(K_{\widetilde{Z}}^{\vee} \otimes S, K_{\widetilde{Z}} \otimes S\right)^{\vee} \rightarrow \operatorname{Ext}_{\mathcal{O}_{\widetilde{Z}}}^{1}(S, S) \times \operatorname{Ext}_{\mathcal{O}_{\widetilde{Z}}}^{1}(S, S) .
$$

Denote the support of $S$ by $\widetilde{\Sigma}$; by assumption, this is a smooth connected curve.

Claim 5.3. The space

$$
\operatorname{Ext}_{\mathcal{O}_{\widetilde{Z}}}^{2}\left(K_{\widetilde{Z}}^{\vee} \otimes S, K_{\widetilde{Z}} \otimes S\right)^{\vee}
$$

admits a subspace isomorphic to $H^{0}(\widetilde{\Sigma}, \mathbf{C})$.

Proof. This is a standard application of the spectral sequence abutting to $\operatorname{Ext}_{\mathcal{O}_{\widetilde{Z}}}^{2}$ and of Serre duality on $\widetilde{\Sigma}$, making use of the identification

$$
N_{\widetilde{\Sigma} \mid \widetilde{Z}} \cong K_{\widetilde{\Sigma}}\left(-\widetilde{\Sigma} \cap D_{\infty}\right)
$$

provided by the Poisson structure of $\widetilde{Z}$. For details see Lemma 5.3 [24].

The Poisson bivector field $\Pi_{\mathrm{Pic}^{0}}$ on (54) is then defined as the image under (55) of the canonical generator

$$
1 \in H^{0}(\widetilde{\Sigma}, \mathbf{C}) \subset \operatorname{Ext}_{\mathcal{O}_{\widetilde{Z}}}^{2}\left(K_{\widetilde{Z}}^{\vee} \otimes S, K_{\widetilde{Z}} \otimes S\right)^{\vee} .
$$

As it is shown in [10], its symplectic leaves are obtained by fixing the intersection of the support of the sheaf with the degeneracy divisor (52). Given that the curves in the Hilbert scheme satisfy conditions (1)-(5) of Theorem 
4.3, the symplectic leaves are thus obtained by fixing the intersection of the support curve with the exceptional divisors $E_{i, j, j^{\prime}, \ldots, j^{\left(m_{i}-1\right)}}$.

We will need a generalization of the setup of the previous paragraph to a relative situation. Namely, for the parameter space $\mathbf{C}^{r \sum_{i}\left(m_{i}+1\right)}$ of (45) the product

$$
\mathbf{C}^{r \sum_{i}\left(m_{i}+1\right)} \times Z
$$

contains a tautological flat reduced subscheme $\mathcal{C}$ of relative dimension 0 over $\mathbf{C}^{r \sum_{i}\left(m_{i}+1\right)}$ given by

$\mathcal{C}=\left(\left(\zeta_{i, j}, \ldots, \zeta_{i, j, j^{\prime} \ldots, j^{\left(m_{i}\right)}}, \lambda_{i, j, j^{\prime} \ldots, j^{\left(m_{i}\right)}}\right)_{i, j, j^{\prime} \ldots, j^{\left(m_{i}\right)}, \cup_{i} \mathbf{C}\left[\left[z_{i}\right]\right] / I_{\zeta_{i, j}, \ldots, \zeta_{i, j, j^{\prime} \ldots, j}\left(m_{i}-1\right), \lambda} \lambda_{i, j, j^{\prime} \ldots, j}\left(m_{i}\right)}\right)$

where

$I_{\zeta_{i, j}, \ldots, \zeta_{i, j, j^{\prime} \ldots, j}\left(m_{i}-1\right)}=\left(z_{i}, \zeta_{i}-\left(\zeta_{i, j}+z_{i} \zeta_{i, j, j^{\prime}}+\cdots+z_{i}^{m_{i}-1} \zeta_{i, j, j^{\prime} \ldots, j^{\left(m_{i}-1\right)}}+z_{i}^{m_{i}} \lambda_{i, j, j^{\prime} \ldots, j^{\left(m_{i}\right.}}\right)\right)$

in the local chart of $Z$ over a neighborhood of $p_{i}$ with coordinates $z_{i}, \zeta_{i}$ (14). Let us denote by

$$
\widetilde{\mathcal{Z}} \rightarrow \mathbf{C}^{r \sum_{i}\left(m_{i}+1\right)} \times Z
$$

the blow-up of $\mathcal{C}$ in (56). We are interested in the relative Hilbert scheme

$$
\operatorname{Hilb}_{\mathrm{rel}}\left(\widetilde{\mathcal{Z}}, \mathbf{C}^{r \sum_{i}\left(m_{i}+1\right)}, H\right)
$$

of $\widetilde{\mathcal{Z}}$ with respect to $\mathbf{C}^{r \sum_{i}\left(m_{i}+1\right)}$, with Hilbert polynomial on the fibres equal to $H$ given in the previous paragraph. It admits a dense open subscheme

$$
\operatorname{Hilb}_{\text {rel }}^{0}\left(\widetilde{\mathcal{Z}}, \mathbf{C}^{r \sum_{i}\left(m_{i}+1\right)}, H\right)
$$

parameterizing smooth connected curves over $\mathbf{C}^{r \sum_{i}\left(m_{i}+1\right)}$. Indeed, let $A \subset$ $\mathrm{C}^{r \sum_{i}\left(m_{i}+1\right)}$ be the constructible subset consisting of nested sequences of eigenvalues for which the eigenvalues $\lambda_{i, j, j^{\prime} \ldots, j\left(m_{i}\right)}$ of the residue at $p_{i}$ restricted to common eigenspaces of the irregular part are of multiplicity 1 . Then, away from $A$ the curves in (57) are smooth over $D_{\text {red, }}$ and in the fibers of (57) over points of $\mathbf{C}^{r \sum_{i}\left(m_{i}+1\right)} \backslash A$ further Zariski open subsets parametrize curves that are everywhere smooth. Notice also that the dimensions $d_{i, j, j^{\prime}, \ldots, j^{\left(m_{i}-1\right)}}$ may change in a discrete way giving different Hilbert polynomials, and this splits up the relative Hilbert scheme into several components. In the Dolbeault setup, this corresponds to letting the dimensions of the joint eigenspaces of the matrices in the irregular part vary.

Finally, we are interested in

$$
\operatorname{Pic}_{\mathrm{rel}}\left(\widetilde{\mathcal{Z}}, \mathbf{C}^{r \sum_{i}\left(m_{i}+1\right)}, H, d\right)
$$

parameterizing torsion-free sheaves of given degree $d$ on the curves in (57). It admits a dense open subscheme

$$
\operatorname{Pic}_{\text {rel }}^{0}\left(\widetilde{\mathcal{Z}}, \mathbf{C}^{r \sum_{i}\left(m_{i}+1\right)}, H, d\right) \rightarrow \operatorname{Hilb}_{\text {rel }}^{0}\left(\widetilde{\mathcal{Z}}, \mathbf{C}^{r \sum_{i}\left(m_{i}+1\right)}, H\right)
$$


parameterizing line bundles supported on a family of smooth connected curves over $\mathbf{C}^{r \sum_{i}\left(m_{i}+1\right)}$.

The Poisson bivector field $\Pi_{\mathrm{Pic}^{0}}$ on (54) described after Claim 5.3 admits a straightforward extension to (60) for which the natural map to $\mathbf{C}^{r \sum_{i}\left(m_{i}+1\right)}$ consists of Casimir operators, simply by pushing forward bivectors. Namely, the inclusion

$$
\widetilde{Z} \hookrightarrow \widetilde{\mathcal{Z}}
$$

gives rise to a map of cotangent bundles

$$
T^{*} \operatorname{Pic}_{\text {rel }}^{0}\left(\widetilde{\mathcal{Z}}, \mathbf{C}^{r \sum_{i}\left(m_{i}+1\right)}, H, d\right) \rightarrow T^{*} \operatorname{Pic}_{\text {rel }}^{0}(\widetilde{Z}, H, d)
$$

and the Poisson structure on (60) is then defined as the composition

$$
\begin{gathered}
T^{*} \operatorname{Pic}_{\mathrm{rel}}^{0}\left(\widetilde{\mathcal{Z}}, \mathbf{C}^{r \sum_{i}\left(m_{i}+1\right)}, H, d\right) \wedge T^{*} \operatorname{Pic}_{\mathrm{rel}}^{0}\left(\widetilde{\mathcal{Z}}, \mathbf{C}^{r \sum_{i}\left(m_{i}+1\right)}, H, d\right) \rightarrow \\
\rightarrow T^{*} \operatorname{Pic}_{\mathrm{rel}}^{0}(\widetilde{Z}, H, d) \wedge T^{*} \operatorname{Pic}_{\mathrm{rel}}^{0}(\widetilde{Z}, H, d) \stackrel{\Pi_{\mathrm{Pic}} 0}{\longrightarrow} \mathbf{C} .
\end{gathered}
$$

Let us define

$$
\mathcal{M}_{\mathrm{Dol}}^{\mathrm{irr}, 0} \subseteq \mathcal{M}_{\mathrm{Dol}}^{\mathrm{irr}}
$$

to consist of equivalence classes of Higgs bundles with smooth connected spectral curve $\widetilde{\Sigma}$, unramified over $D_{\text {red }}$. With this notation the following result holds.

Theorem 5.4. The equivalence of categories of Theorem 4.3 induces a Poisson isomorphism between dense open subets of the spaces (45) and (59):

$$
\begin{aligned}
\left(\mathcal{M}_{\text {Dol }}^{\mathrm{irr}, 0}, \Pi_{\text {Dol }}\right) & \cong\left(\operatorname{Pic}_{\mathrm{rel}}^{0}\left(\widetilde{\mathcal{Z}}, \mathbf{C}^{r \sum_{i}\left(m_{i}+1\right)}, H, d\right), \Pi_{\text {Pic }}\right), \\
d & =\delta+\frac{r(r-1)}{2} \operatorname{deg}(L)
\end{aligned}
$$

with $\delta$ defined in Remark 5.1.

Proof. First let us observe that by definition for $(\mathcal{E}, \theta) \in \mathcal{M}_{\text {Dol }}^{\text {irr, } 0}$ the eigenspaces of the residue of $\theta$ are 1-dimensional. Therefore, the choice of a parabolic filtration on the eigenspaces is vacuous, so Theorem 4.3 indeed identifies $\mathcal{M}_{\text {Dol }}^{\text {irr, }}$ with (60).

It is now sufficient to show that the symplectic structures on the symplectic leaves get identified. This is precisely the content of the key observation (24) of Proposition 5.1 of [24] (c.f. also [11] Proposition 2.30 in the case of the holomorphically trivial vector bundle over the projective line). The formula for $d$ follows directly from the well-known fact that the direct image of $\mathcal{O}_{\Sigma}$ is equal to

$$
\mathcal{O}_{C} \oplus L^{-1} \oplus \cdots \oplus L^{1-r}
$$


SZILÁRD SZABÓ

\section{ACKNOWLEDGMENTS}

This paper grew out of work carried out under the support of the Advanced Grant "Arithmetic and physics of Higgs moduli spaces" no. 320593 of the European Research Council and the Lendület "Low Dimensional Topology" program of the Hungarian Academy of Sciences. The author would like to thank the École Polytechnique Fédérale de Lausanne and the Alfréd Rényi Institute of Mathematics for their hospitality.

\section{REFERENCES}

[1] Kürşat Aker and Szilárd Szabó. Algebraic Nahm transform for parabolic Higgs bundles on $\mathbf{P}^{1}$. Geometry and Topology, 18(5):2487-2545, 2014.

[2] Michi aki Inaba and Masa-Hiko Saito. Moduli of unramified irregular singular parabolic connections on a smooth projective curve. Kyoto J. Math., 53(2):433-482, 2013.

[3] Allen B. Altman and Steven L. Kleiman. Compactifying the Picard scheme. Advances in Mathematics, 35:50-112, 1980.

[4] Arnaud Beauville, M. S. Narasimhan, and S. Ramanan. Spectral curves and the generalised theta divisor. J. reine angew. Math., 398:169-179, 1989.

[5] Olivier Biquard and Philip Boalch. Wild non-abelian Hodge theory on curves. Compos. Math., 140(1):179-204, 2004.

[6] Indranil Biswas and Sundararaman Ramanan. An infinitesimal study of the moduli of Hitchin pairs. J. London Math. Soc., 49(2):219-231, 1994.

[7] Philip P. Boalch. Hyperkaehler manifolds and nonabelian Hodge theory of (irregular) curves. arXiv:1203.6607.

[8] Philip P. Boalch. Geometry and braiding of Stokes data; fission and wild character varieties. Ann. of Math., 179(1):301-365, 2014.

[9] Hans U. Boden and Kôji Yokogawa. Moduli spaces of parabolic Higgs bundles and parabolic K(D) pairs over smooth curves. I. Internat. J. Math., 7(5):573-598, 1996.

[10] Ron Donagi and Eyal Markman. Spectral covers, algebraically completely integrable Hamiltonian systems, and moduli of bundles, volume 1620 of Lecture Notes in Math. Springer, 1996.

[11] John Harnad and Jacques Hurtubise. Multi-Hamiltonian structures for r-matrix systems. J. Math. Phys., 49(6), 2008.

[12] Nigel J. Hitchin. Stable bundles and integrable systems. Duke Math. J., 54:91-114, 1987.

[13] Jacques C. Hurtubise. Integrable systems and algebraic surfaces. Duke Math. J., 83(1):19-50, 1996.

[14] Daniel Huybrechts and Manfred Lehn. The geometry of moduli spaces of sheaves. Cambridge University Press, 2010.

[15] Hiroshi Konno. Construction of the moduli space of stable parabolic Higgs bundles on a Riemann surface. J. Math. Soc. Japan, 45(2):253-276, 1993.

[16] Maxim Kontsevich and Yan Soibelman. Wall-crossing structures in DonaldsonThomas invariants, integrable systems and mirror symmetry. arXiv:1303.3253.

[17] Marina Logares and Johan Martens. Moduli of parabolic Higgs bundles and Atiyah algebroids. J. reine angew. Math., 649:89116, 2010. 
[18] Eyal Markman. Spectral curves and integrable systems. Compos. Math., 93(3):255290, 1994.

[19] V. B. Mehta and C. S. Seshadri. Moduli of vector bundles on curves with parabolic structures. Math. Ann., 248:205-239, 1980.

[20] Takuro Mochizuki. Wild harmonic bundles and wild pure twistor D-modules. Number 340 in Astérisque. Société Mathématique de France, 2011.

[21] Daniel Schaub. Courbes spectrales et compactifications de jacobiennes. Math. Z., 227:295-312, 1998.

[22] Carlos T. Simpson. Harmonic bundles on noncompact curves. J. Amer. Math. Soc., 3(3):713-770, 1990.

[23] Szilárd Szabó. Nahm transform for integrable connections on the Riemann sphere. Mémoires de la Société Mathématique de France, 110:1-114, 2007.

[24] Szilárd Szabó. The Plancherel theorem for Fourier-Laplace-Nahm transform for connections on the projective line. Communications in Mathematical Physics, 338(2):753-769, 2015.

[25] Edward Witten. Gauge theory and wild ramification. Anal. Appl. (Singap.), 6(4):429$501,2008$.

[26] Kôji Yokogawa. Compactification of moduli of parabolic sheaves and moduli of parabolic Higgs sheaves. J. Math. Kyoto Univ., 33(2):451-504, 1993. 